\title{
Modeling mass-dependent flow regime transitions to predict the stopping and depositional behavior of snow avalanches
}

\author{
P. Bartelt, ${ }^{1}$ Y. Bühler, ${ }^{1}$ O. Buser, ${ }^{1}$ M. Christen, ${ }^{1}$ and L. Meier ${ }^{1}$ \\ Received 23 December 2010; revised 5 November 2011; accepted 7 November 2011; published 22 February 2012.
}

[1] How terrain, snow cover properties, and release conditions combine to determine avalanche speed and runout remains the central problem in avalanche science. Here we report on efforts to understand how surface roughness, snow properties, and internal mass fluxes control the generation of granular fluctuation energy within the basal shear layers of dense flowing snow avalanches, and the subsequent influence on avalanche speed and deposition patterns. For this purpose we augment the depth-averaged equations of motion to account for the generation of the kinetic energy associated with the particle fluctuations, and dissipation of this energy by collisional and frictional material interactions. Using high-resolution laser scans of the preevent snow cover and postevent deposits from two avalanches released at the Swiss Vallée de la Sionne observation station, we compare measured and calculated deposition heights. The model captures flow velocities and deposition heights without ad hoc adjustments of the constitutive parameters according to avalanche size. The model parameters are separated into a terrain and other pure material (snow) parameters. The investigations reveal how release conditions and snow entrainment influence the internal mass distribution, and control flow regime transitions between the fluidized regime (head) and plug regime (tail). The comparison between the measured and calculated velocities and deposition heights demonstrates why standard Voellmy-type submodels are suitable for many practical mitigation problems, but also why they are limited to cases where the calibrated parameters, already accounting for terrain, snow cover properties, avalanche size, and mass intake, are known.

Citation: Bartelt, P., Y. Bühler, O. Buser, M. Christen, and L. Meier (2012), Modeling mass-dependent flow regime transitions to predict the stopping and depositional behavior of snow avalanches, J. Geophys. Res., 117, F01015, doi:10.1029/2010JF001957.

\section{Introduction}

[2] It is the purpose of this paper to present an avalanche dynamics model that explicitly accounts for the granular behavior of flowing snow. As soon as the snow cover sets in motion, it fragments and breaks up into collision-hardened particles of various shapes and sizes (median particle sizes range between $70 \mathrm{~mm}$ and $100 \mathrm{~mm}$ [Jomelli and Bertran, 2001; Bartelt and McArdell, 2009]). Depending on terrain and the material properties of the particles, the granular fluid can quickly reach velocities of more than $50 \mathrm{~m} / \mathrm{s}$. The granular interactions are collisional and frictional: the particles collide and fracture under impact, but likewise they slide, scrape and wear under continual contact. The diverse array of particle interactions produces a wide variety of flow behavior depending on particle properties (size, hardness, dry or wet) and terrain characteristics, which control the particle interactions with the snow cover and ground. Snow avalanches can therefore display different flow forms

\footnotetext{
${ }^{1}$ WSL Institute for Snow and Avalanche Research SLF, Davos, Switzerland.

Published in 2012 by the American Geophysical Union.
}

ranging from heavy dense flowing avalanches to powder suspension currents, and all the transitional and mixed avalanche forms [Simpson, 2010]. These flow forms are reflected in the large-scale deposition features of the avalanches at rest [Issler and Gauer, 2008], as well as in the dimensions of the particles found in avalanche deposits [Bartelt and McArdell, 2009].

[3] Although in theory it would be possible to trace the trajectories of millions of interacting particles in order to model real avalanches, there is little practical incentive to do so. The calculation demands are enormous and the exact knowledge of all of the particle positions and velocities is simply of no engineering interest. Avalanche science is primarily concerned with finding a reduced description of avalanche flow that allows a pragmatic, macroscopic modeling of avalanches in real terrain. This is similar to the thermodynamic treatment of gases, where individual molecular movements are not considered, but are reduced to three continuum state variables: pressure, volume and temperature, which can be measured and compared to model predictions [Glansdorff and Prigogine, 1977]. A reduced avalanche description is also in good accordance with our present experimental capabilities to measure macroscopic state variables: leading edge and internal avalanche velocities [Gauer 

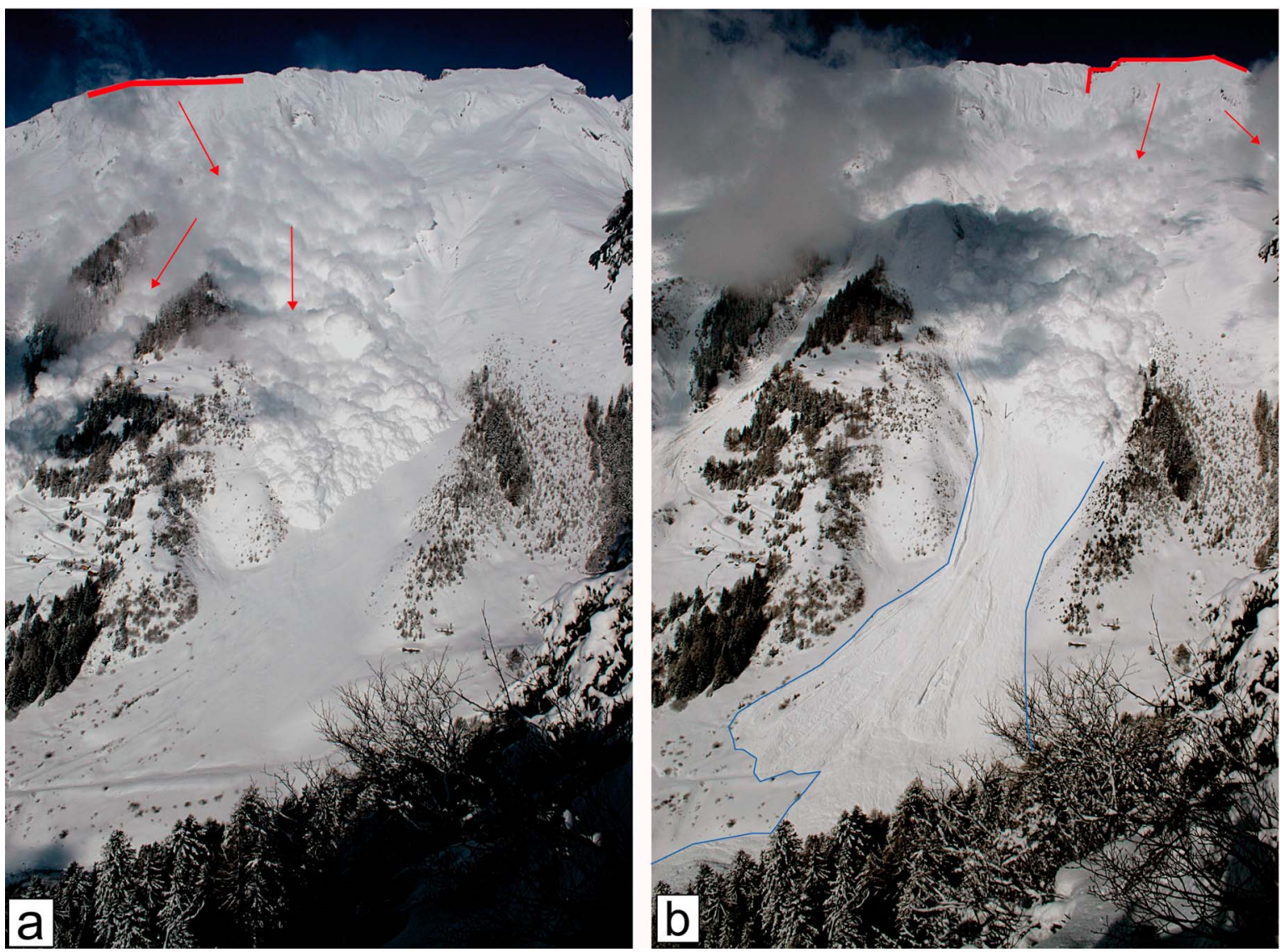

Figure 1. Vallée de la Sionne (VdlS) avalanches of 6 March 2006. (a) Avalanche 816 originating from the Crêta Besse 1 (CB1) release zone. The avalanche descended fastest on the left hand side of the picture, hugging the channel side. The avalanche spread laterally over the runout zone. Note the undisturbed snow cover encountered by the avalanche. (b) Avalanche 817 started from Crêta Besse 2 (CB2) and ran over the deposits of avalanche 816. Note the widespread distribution of the avalanche deposits from avalanche 816 . Much of the avalanche stopped in the transition zone leading up to the counterslope. The cloud overhanging the VdlS slope prevented us from determining the leading edge velocities of avalanche 817 in the upper regions of the track.

et al., 2007a, 2007b, 2009; Kern et al., 2009, 2010], energy [Vilajosana et al., 2007], basal shear and normal pressures [Platzer et al., 2007], in addition to flow and deposition heights [Sovilla et al., 2010]. Even if we did calculate trajectories, we would have little experimental information from real-scale avalanches to judge the correctness of the particle calculations.

[4] In this paper we present a reduced description of avalanche flow that takes into account the role of the kinetic energy associated with the particle fluctuations [Bartelt et al., 2006; Buser and Bartelt, 2009; Christen et al., 2010a]. The granular properties of avalanches have long been recognized and are implicitly accounted for in blocktype avalanche models [Perla et al., 1980; Salm et al., 1990], flowing avalanche models [Savage and Hutter, 1989, 1991; Hungr, 1995; Volk and Kleemayr, 1999; Naaim et al., 2003, 2004; Gruber and Bartelt, 2007] and powder snow avalanche models [Fukushima and Parker, 1990; Ancey, 2004; Sampl and Zwinger, 2004; Turnbull et al., 2007]. In these approaches, different frictional relationships are used to model flowing snow(see for example Salm [1993]). Exactly because avalanches do not flow as a single block, it is necessary to define explicit relations governing the production and dissipation of the kinetic energy associated with the particle fluctuations. By accounting for the macroscopic energy fluxes associated with these random movements, we take an initial step toward developing thermodynamically consistent models that allow the treatment of flow regime transitions [Issler and Gauer, 2008; Bartelt et al., 2011], density variations [Buser and Bartelt, 2011] as well as the long-standing frictional problems of avalanche runout and deposition.

[5] The reduced description requires an additional energy equation with closure relations describing how the particle fluctuations influence the frictional stress and therefore the avalanche velocity. The energy equation describes how the fluctuations are created from the mean flow and how the fluctuations decay in time. To verify these concepts comparison of model results with measurements is essential. We compare calculated flow velocities and deposition heights with measurements of avalanches 816 and 817 artificially released at the Vallée de la Sionne (VdlS) test site on 6 March 2006 (Figure 1). We show that we are able to model the avalanche front velocity, controlled by the increase of 

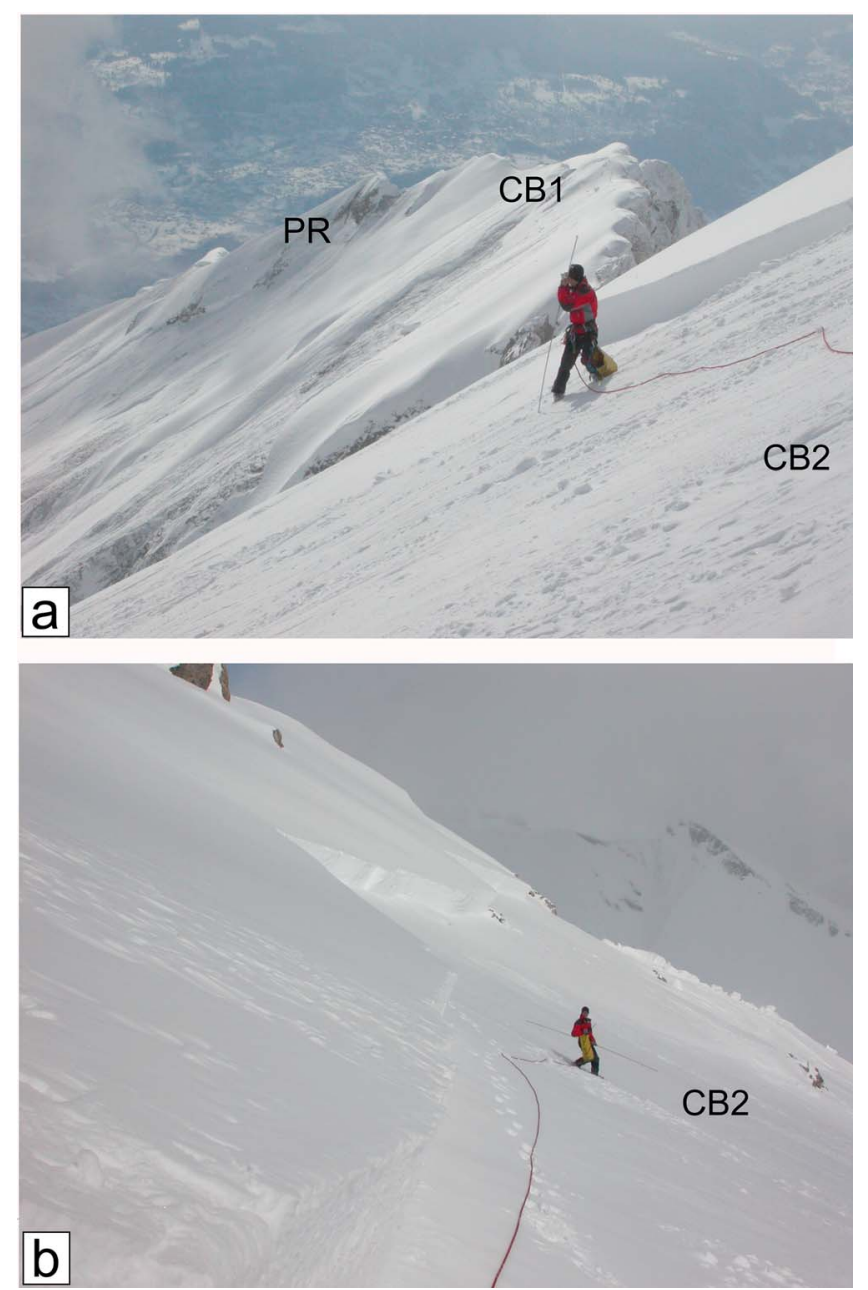

Figure 2. Avalanche release zones. (a) The Pra Roua (PR) and Crêta Besse 1 (CB1) release zones of avalanches 816 are located in the distance of the top photograph. The picture was taken from release zone Crêta Besse 2 (CB2). No direct measurements were performed in CB1. (b) Fracture crown of avalanche 817 (CB2). The fracture crown extended $400 \mathrm{~m}$ along the Crêta Besse ridge.

particle fluctuation energy, while likewise capturing the deposition of the avalanche tail, which we find is controlled by the waning production of particle fluctuation energy [Bartelt et al., 2007]. Snow avalanches have different flow regimes at the front and at the tail suggesting a positiondependent frictional component that cannot be modeled with frictional relationships that implicitly account for the random particle movements. This fact explains why engineers often require different friction parameter sets to model avalanches of different size and type [Gruber and Bartelt, 2007]. The position-dependent frictional component produces unique avalanche deposition patterns that can be measured and compared to simulation results. The comparison reveals why avalanche size and avalanche mobility are intimately linked and why in future it might be possible to find constitutive constants based only on the material properties of the snow granules and track properties such as surface roughness and vegetation. To underscore the influence of mass on avalanche mobility we additionally simulate the smaller VdlS avalanche 917 (26 March 2009) with the same material constants, again using frictional parameters depending on the position-dependent particle fluctuation energy. We begin with a description of the full-scale experiments.

\section{Observations}

\subsection{VdIS Test Site}

[6] The Swiss Vallée de la Sionne observation station was constructed in 1997 to facilitate direct measurements on fullscale snow avalanches. The site was partially destroyed in 1999 by three catastrophic avalanches and subsequently rebuilt in 2001. Since the winter of 2002-2003 measurements on several spontaneous and artificially released avalanches have again been successfully recorded.

[7] The site is located in Canton Valais, $7.5 \mathrm{~km}$ North of the town Sion $\left(7^{\circ} 22^{\prime} 02.28^{\prime \prime}\right.$ E $\left.46^{\circ} 17^{\prime} 55.32^{\prime \prime} \mathrm{N}\right)$. The $2700 \mathrm{~m}$ long avalanche track (Figure 1) is situated on the southeast facing slope of the Crêta Besse $(2650 \mathrm{~m})$. The avalanche track drops in total $1300 \mathrm{~m}$ to the valley bottom where the small mountain creek, La Sionne, separates the long deceleration zone and the counterslope. A manned, concrete observation bunker is situated on the counterslope. From here, the three primary release zones located between $2,300 \mathrm{~m}$ and $2,600 \mathrm{~m}$ above sea level can be directly observed: Pra Roua (PR, average slope $36.5^{\circ} \pm 4.0^{\circ}$ ), Crêta Besse $1\left(\mathrm{CB} 1\right.$, average slope $\left.40.4^{\circ} \pm 5.5^{\circ}\right)$ and Crêta Besse $2\left(\mathrm{CB} 2\right.$, average slope $36.2^{\circ} \pm 3.9^{\circ}$ ) (Figure 2). Release volumes from each of these three zones can reach $100,000 \mathrm{~m}^{3}$. Avalanches from PR and CB1 descend through a hilly terrain before entering the orographic right flow gully of the VdlS track; avalanches from CB2 flow into the orographic left VdlS gully. Both flow gullies are $100 \mathrm{~m}$ wide, approximately $1750 \mathrm{~m}$ long and have an average slope of $36.1^{\circ}$ (Figure 3 ). The gullies exit into a flat, open flow plane where a $20 \mathrm{~m}$ high tubular steel pylon stands $(1640 \mathrm{~m})$. The pylon is among other sensors instrumented with optical velocity sensors to determine the internal velocity profiles of the avalanches [Kern et al., 2009, 2010]. The pylon defines the beginning of a $560 \mathrm{~m}$ long transition/deceleration zone (average slope $19.1^{\circ} \pm 2.0^{\circ}$ ) which an avalanche must cross before striking the steep, $170 \mathrm{~m}$ long counter slope (average slope $40^{\circ}$ below the bunker). Large avalanches are deflected by the counter slope and run in the downstream direction of the La Sionne. The VdlS track surface is composed of exposed bedrock and small grain mountain scree interlaced with grass tussocks and the occasional bush. The counterslope below the bunker is covered with thick stands of green alder (Alnus veridus) and rowan (Sorbus aucuparia); a spruce forest (Picea abies), that was partially destroyed by the extreme avalanches of 1999 , is located directly above the bunker.

\subsection{Avalanche 816}

[8] The first avalanche on 6 March 2006 was artificially released (one charge) at 10:00 (Figure 1a). It started from the Crêta Besse 1 (CB 1) and Pra Roua (PR) release zones (Figure 2a). Two separate, but closely situated, slabs released simultaneously with an estimated total volume of $V_{0}=103,000 \mathrm{~m}^{3}$. The aerial laser scanning measurements revealed that the average fracture height of the PR zone was larger $\left(h_{0}=1.5 \mathrm{~m}\right)$ than the CB1 zone $\left(h_{0}=1.0 \mathrm{~m}\right)$, 


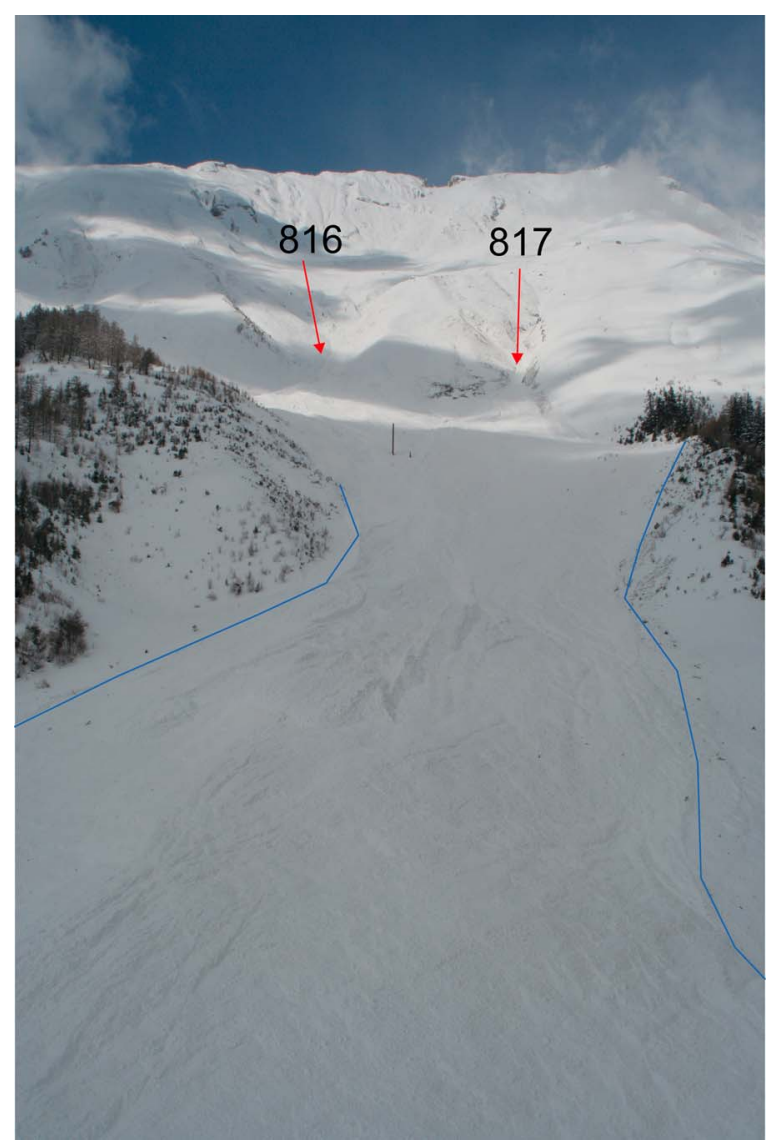

Figure 3. Photograph of the acceleration zone of the VdlS avalanche site taken from the counterslope directly after avalanches 816 and 817. Avalanche deposits from avalanche 817 covered the entire runout zone. Entrainment scars are visible. The $20 \mathrm{~m}$ high pylon is located in the middle of the picture. Primary flow channels of both avalanches are defined. Note the uneven structure of the deposits.

indicating a large variability in snow cover distribution. Most of the released mass entered the orographic right VdlS gully; however, some mass from the PR release zone fed a secondary channel, Incron, running parallel to the main track (Figure 4). Both avalanches in the primary and secondary tracks reached the valley bottom. The mass in the main channel accelerated quickly, reaching peak front velocities of $50 \mathrm{~m} / \mathrm{s}$ at the measurement pylon (Figure 5). Photographs show exposed ground surfaces, suggesting considerable snow cover entrainment (Figure 3). Unfortunately, no pre or postevent laser scanning was performed in the upper regions of the 816 track where entrainment occurred. The nose of the avalanche passed to the orographic right hand side of the measurement pylon (Figure 1); optical velocity sensors on the pylon measured the internal velocity distribution [Kern et al., 2009]. The maximum measured internal velocities are approximately $35 \mathrm{~m} / \mathrm{s}$, lower than maximum leading edge velocity. The avalanche struck the counter slope, depositing mass on the measurement bunker (Figure 4). Avalanche deposits covered the transition zone, extending between the measurement pylon and the La Sionne creek. Some of the avalanche mass, after striking the counterslope, turned and moved down the La Sionne gully.
We estimate the avalanche deposits in this region to be between $5 \mathrm{~m}$ and $10 \mathrm{~m}$. A particularly distinctive feature of the deposits of this avalanche is the flow arm that bent toward the orographic right hand side of the main VdlS runout zone. This arm is visible in the photograph of avalanche 817 (Figure 1b).

\subsection{Avalanche 817}

[9] The second avalanche was artificially released (one charge) at 10:50 from the Crêta Besse 2 (CB2) release zone, 50 minutes after avalanche 816. (Time is required to download data which is stored locally at the sensors.) The fracture crown extended $400 \mathrm{~m}$ along the CB2 ridge (Figure $2 \mathrm{~b}$ ). The mean fracture height, obtained from the laser scanning measurements, was $h_{0}=1.3 \mathrm{~m}$. The estimated total release volume of $V_{0}=119,000 \mathrm{~m}^{3}$ was therefore slightly larger than avalanche 816 . However, some of the release mass fed the Tsarmettes avalanche track (Figure 4). The main avalanche body accelerated rapidly, especially in the steep gully leading to the VdlS transition zone. Measured front propagation velocities were approximately $55 \mathrm{~m} / \mathrm{s}$. The laser scanning measurements reveal that at some locations in the channel over $3 \mathrm{~m}$ of snow was eroded by the avalanche. The avalanche ran to the orographic left hand side of the measurement pylon (Figure 1) and the signals from the optical velocity sensors could not be correlated because of the oblique impact. Therefore no information regarding the internal velocity distribution is available for this avalanche. In the runout zone the avalanche ran over the deposits of avalanche 816 , covering them to a large extent (Figures $1 \mathrm{~b}$ and 3). Any exposed deposits were either overrun by the dense core of the avalanche or were wind blasted by the powder cloud. The granulometric dimensions of the surface deposits of both avalanches were measured using a sediment size sampling method at three locations in the transition zone [Bartelt and McArdell, 2009]. The median particle diameter was approximately $70 \mathrm{~mm}$ (Figure 6).

\subsection{Laser Scanning Measurements}

[10] Aerial laser scanning measurements were performed before avalanche 816 and after avalanche 817 over the area $A$ (Figure 4) [Sovilla et al., 2010]. This area includes the main flow path of avalanche 817 , but only part of the avalanche 816. Although the entire release area in the upper regions of the Crêta Besse ridge was scanned, the acceleration zone of avalanche 816 was not included in the measurement domain as incoming bad weather and cost prohibited a complete laser scan of the inundated area. The avalanche tracks, Incron and Tsarmettes, could also not be included in the helicopter flight path, even though considerable avalanche mass flowed down these secondary tracks. The transition zone between the mast and counterslope of the main VdlS track, containing the avalanche deposits from both avalanches 816 and 817 , was recorded. In this region it is not possible to separate the deposits of avalanche 816 from the deposits of avalanche 817. The avalanche deposits contained large-scale flow features such as frontal lobe pileup, en echelon type shear faults and sidewall levees.

[11] Let $h_{1}(x, y)$ denote the first (preavalanche) measurement of the vertical snow cover height; let $h_{2}(x, y)$ denote the second (postavalanche) measurement of the vertical deposition height (Figure 7). The coordinate pair $(x, y)$ defines the 


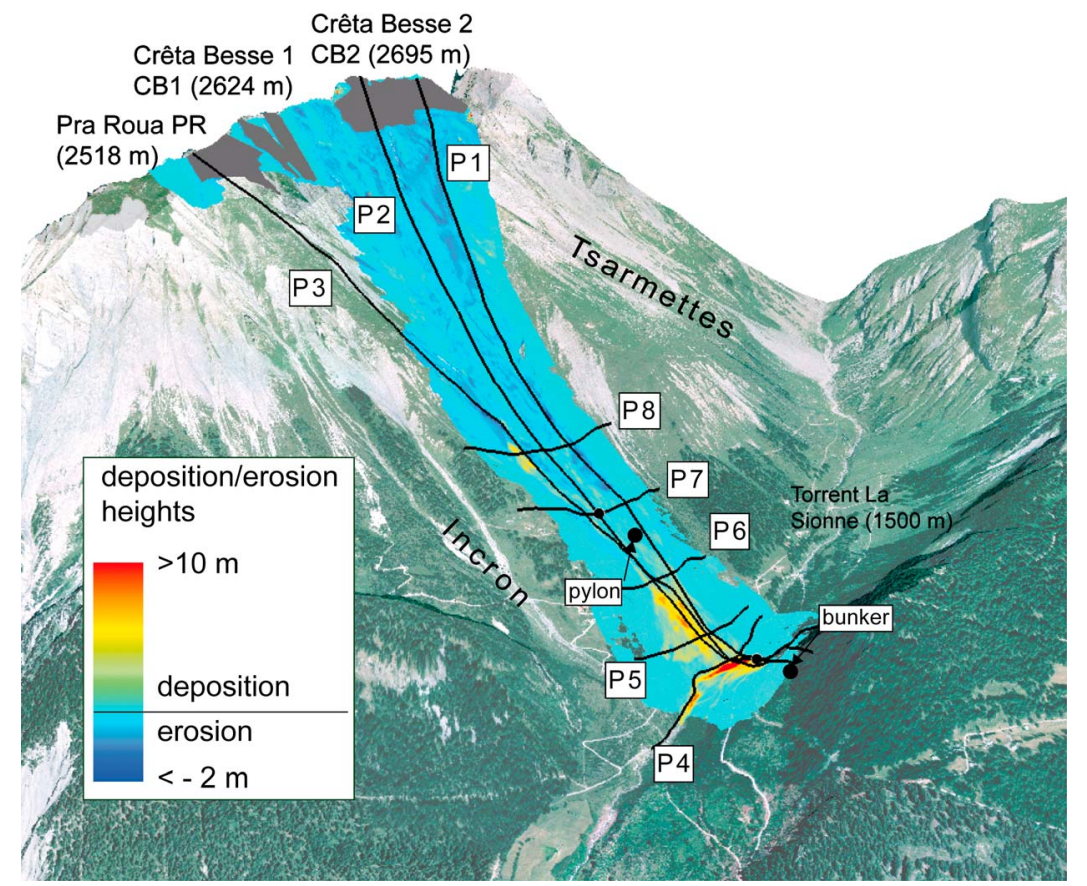

Figure 4. Three dimensional depiction of the Vallée de la Sionne observation station. The plot defines the area $A$ where preavalanche $h_{1}$ and postavalanche $h_{2}$ laser scanning was performed. Colors depict $\Delta h=h_{2}-h_{1}$. We evaluated the laser scanning data on the longitudinal profiles $\mathrm{P} 1-\mathrm{P} 3$ and transversal profiles P4-P8.

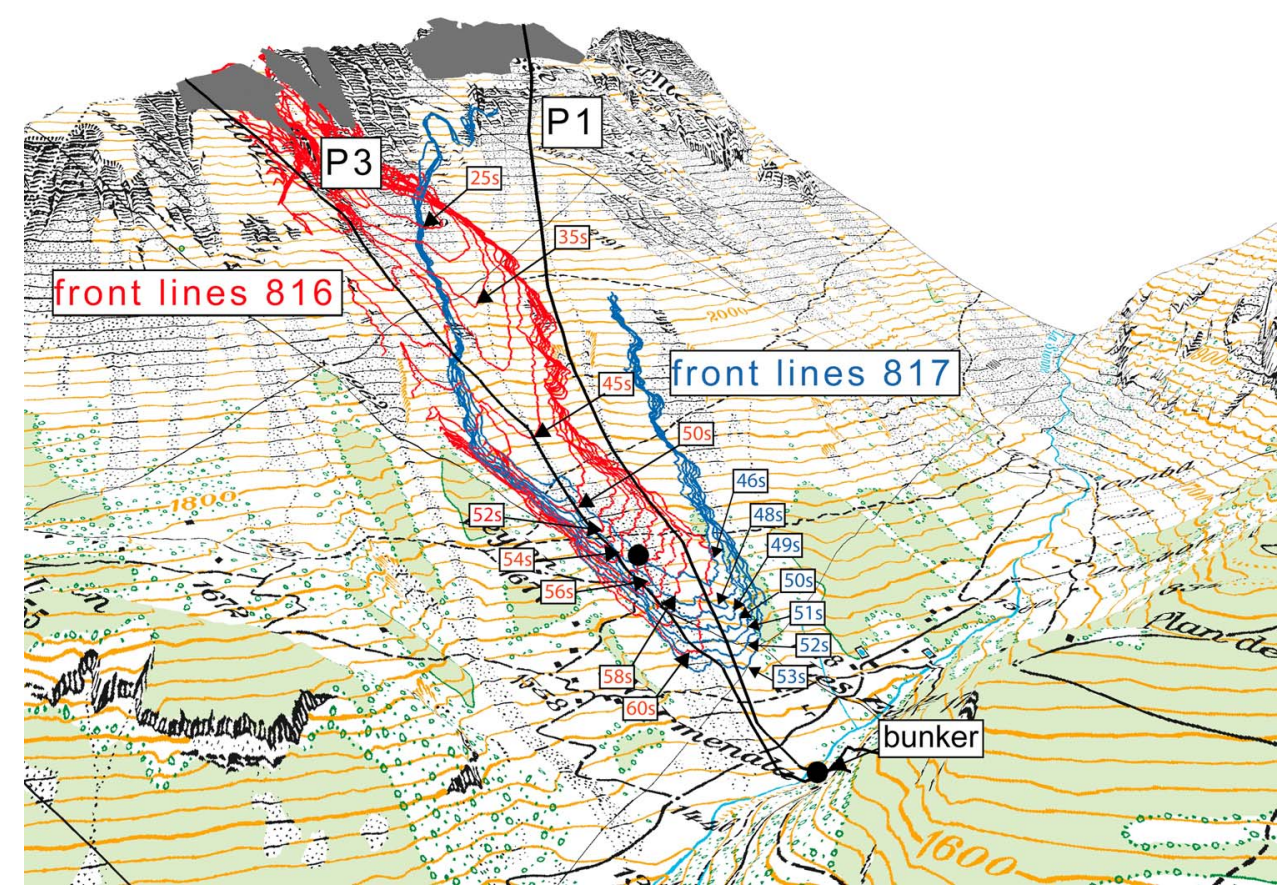

Figure 5. Release zone of avalanches 816 and 817 . Front locations as a function of time are superimposed on the surface for both avalanches 816 (red) and 817 (blue). From georeferenced video recordings of the event we determined the position of the front. Knowing the travel time between front locations we could determine the leading edge velocity. 


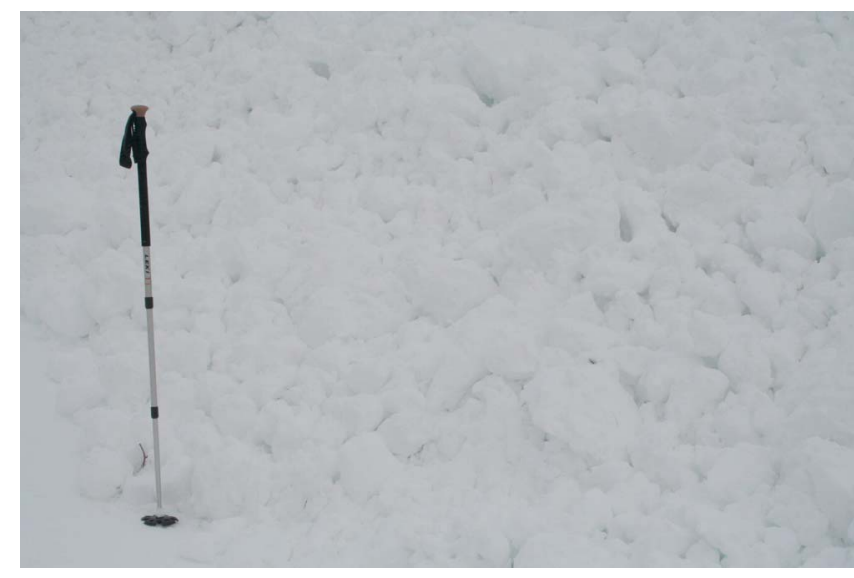

Figure 6. Close-up of the avalanche deposits with a ski pole for scale. See Bartelt and McArdell [2009]. The granular properties of dry snow avalanches are a driving motivation for the random kinetic energy (RKE) model.

location in the surface domain $A$. For a single avalanche, the height distribution of the avalanche deposits $h_{d}(x, y)$ is equal to

$$
h_{d}=h_{2}-h_{1}+h_{e}=\Delta h+h_{e}
$$

where $h_{e}(x, y)$ is the height of the snow cover entrained and eroded by the avalanche and $\Delta h$ is the difference of laser scanning measurements [Sovilla et al., 2010]. Since the running surface of the avalanche is unknown, only the net difference between the deposition and entrainment height $\Delta h=h_{d}-h_{e}$ can be determined from the laser scanning measurements. Both $h_{d}$ and $h_{e}$ vary with position $(x, y)$ (see Figure 7). However, in the release and acceleration zones, where we observed no avalanche deposits $\left(h_{d}=0\right)$, we can determine the fracture heights

$$
h_{0}=-\Delta h
$$

or snow cover entrainment heights

$$
h_{e}=-\Delta h .
$$

This procedure can only be applied in the regions where the avalanches do not overlap. In the transition zone and counterslope, the determination of avalanche deposition and entrainment heights is complicated because we consider the overlapping avalanches. The sum of avalanche deposition heights is

$$
h_{d}=h_{d}^{816}+h_{d}^{817}=h_{2}-h_{1}+h_{s}
$$

where $h_{s}$ is total submerged depth (measured from $h_{1}$ ) of both avalanches. This depth accounts for the rearrangement of the first deposits of avalanche 816 by the second avalanche 817 . It cannot be determined from the pre and postevent laser scans. The value of $h_{s}$ is, however, bounded by the relation $h_{s} \leq h_{1}-z_{0}$ where $z_{0}$ is the terrain elevation.

\subsection{Slope Angle, Track Roughness, and Height Difference $\Delta \boldsymbol{h}$}

[12] We evaluated slope angle, terrain roughness and the net height difference $\Delta h$ on three longitudinal profiles (P1-P3) and five transversal profiles (P4-P8). Profiles P1 and $\mathrm{P} 2$ start from the $\mathrm{CB} 2$ release zone and descend to the orographic left hand side of the measurement pylon, cross the creek La Sionne and end at the bunker (Figure 4). These profiles cover the flow path of avalanche 817. Profile P3 starts between the PR and CB1 release zones, passes the measurement pylon and ends at the bunker. The first transversal profile P4 is located at the La Sionne creek; P5 and P6 cut the transition zone; P7 is located at the pylon; P8 is located above the pylon (Figure 4).

[13] The slope angles of the two main avalanche profiles $\mathrm{P} 1$ and P3 are depicted in Figure 8. The avalanches start in steep $35^{\circ}-40^{\circ}$ terrain. The tracks flatten somewhat before entering another steep track segment, $1000 \mathrm{~m}$ longitudinal distance from the release zones (Figure 8). Here the avalanches accelerate before entering the remarkably constant $20^{\circ}$ transition zone leading to the counterslope. The acceleration zone in the interior of the VdlS track is the primary reason for the high avalanche velocities in the transition zone. Slope angles between $-10^{\circ}$ (counterslope) and $10^{\circ}$ are few and found at the valley bottom where the deposition heights are largest.

[14] Measured height differences as large as $\Delta h=h_{2}-$ $h_{1}=15 \mathrm{~m}$ are found near the river on the counter slope (Figure 9). Significant deposits, sometimes higher than $\Delta h>5 \mathrm{~m}$, are found in the transition zone of profiles $\mathrm{P} 2$ and $\mathrm{P} 3$, along the $600 \mathrm{~m}$ section leading up to the counterslope.

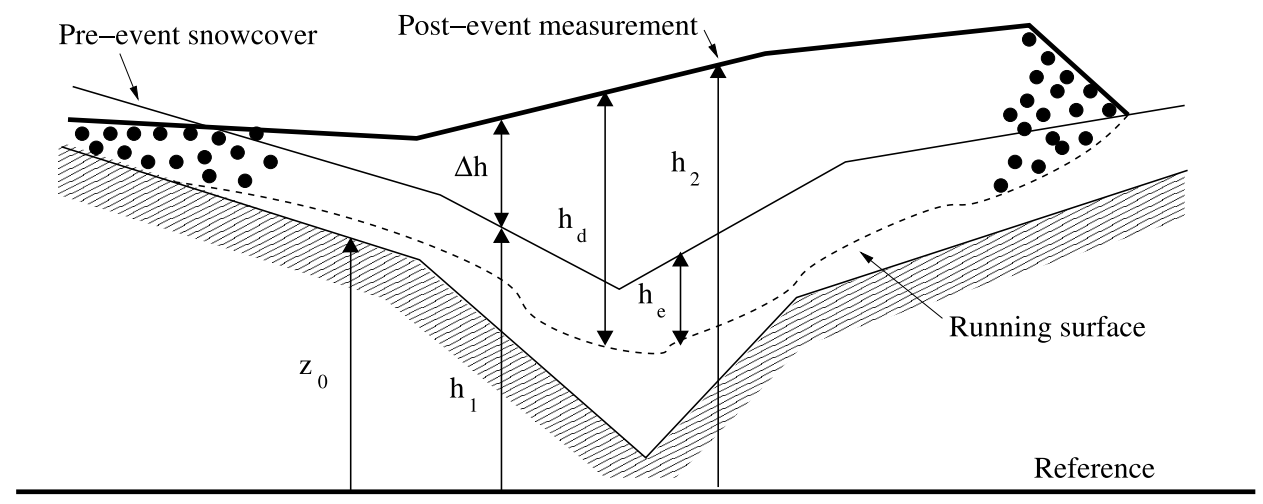

Figure 7. Definition of the vertical $h_{1}$ and $h_{2}$ heights obtained from the aerial laser scanning measurements. The terrain elevation is given by $z_{0}$. 

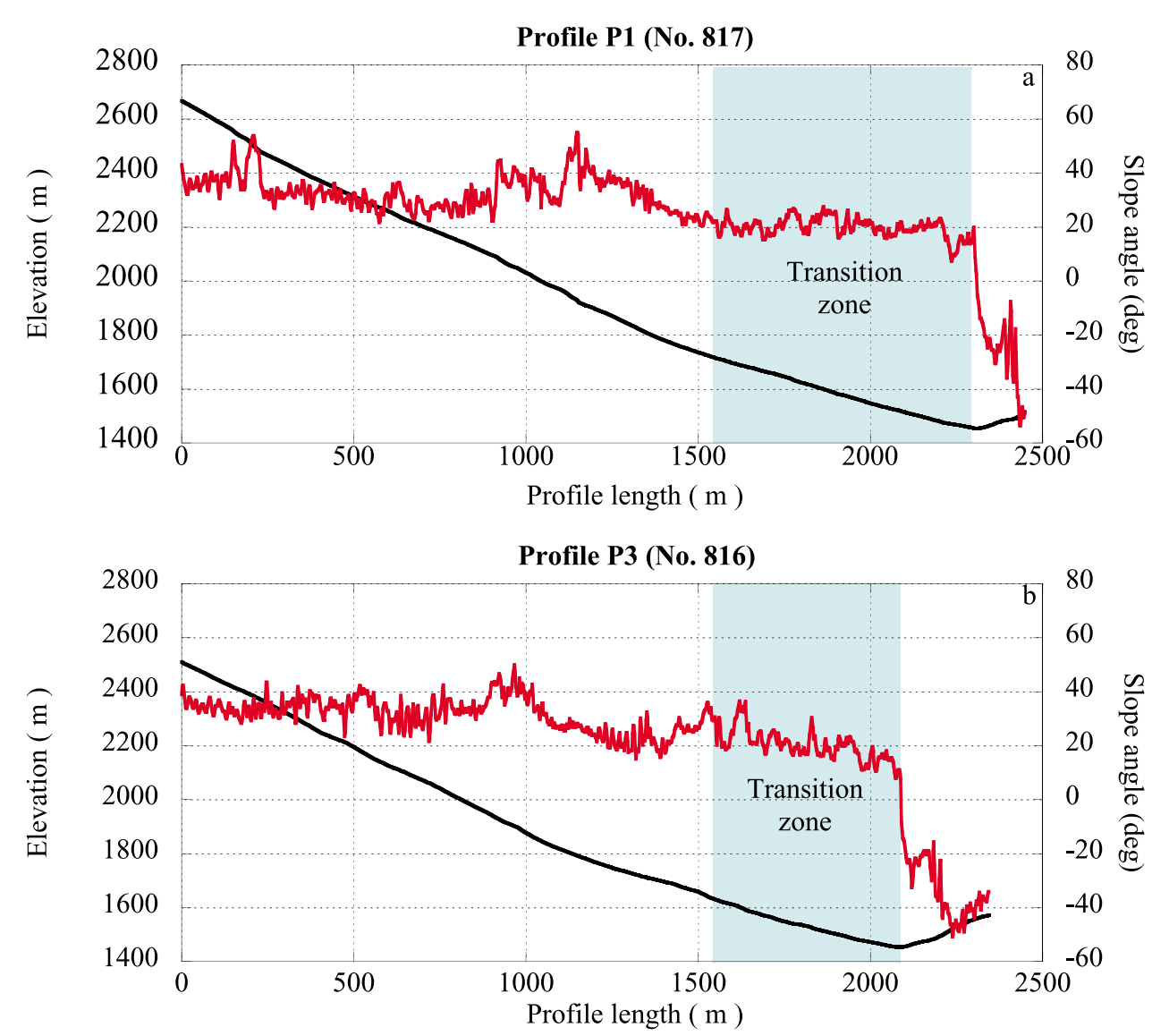

Figure 8. Variation of slope angle along longitudinal profiles (a) P1 (avalanche 817) and (b) P3 (avalanche 816). The profile length is the horizontal distance of the avalanche path. The profile starts at the mountain crest.

As can be expected the measured height difference $\Delta h$ as a function of the profile slope angle shows large scatter (Figure 10). Measured height differences $\Delta h$ can vary between $-2 \mathrm{~m}$ and $7 \mathrm{~m}$ for the same slope angle in the transition zone before the counterslope. The highest deposition heights are located at the negative slope angles because of the counterslope runup. Erosion occurs primarily at slope angles greater than $25^{\circ}$. In the transition zone both erosion and deposition can occur at slope angles between $10^{\circ}$ and $20^{\circ}$. Height differences $\Delta h$ were also recorded on the counterslope. (These are also in good agreement with observations: the bunker was under $2 \mathrm{~m}$ of avalanche snow and a hole had to be dug to reach the bunker entrance.) Along these eight profiles we shall compare the measured and calculated heights obtained from simulating both avalanches 816 and 817.

[15] As a measure of terrain roughness we determined the standard deviation from the mean slope angle using $9 \times 9$ pixel calculation template placed over a summer DEM with $0.5 \mathrm{~m}$ resolution $(4.5 \times 4.5 \mathrm{~m}$ template). The roughness over the entire VdlS track is such that we can call the track smooth (standard deviations of slope angle within the $4.5 \mathrm{~m}$ template less than $1^{\circ}$ ). The counterslope exhibits the largest roughness values; this is because of the steep, cliff-like terrain. The transition zone of avalanche 817 (P1) leading up to the counterslope is both long and smooth (Figure 11a). The transition zone of avalanche 816 is uneven and exhibits some rough segments in the transition zone near the measurement pylon (Figure 11b). The steep track segment in the interior of the avalanche track $(1000 \mathrm{~m}$ from the release zone) exhibits slightly higher roughness values than the rest of the track.

[16] There appears to be no clear relationship between terrain roughness, slope angle and the measured height difference $\Delta h$ on the VdlS track. We present all three values in a plot which combines slope angle, terrain roughness and $\Delta h$ (Figure 12). This plot provides an overview of the depositional pattern for a particular avalanche track. In the transition zone (slope angles near $20^{\circ}$ ) we find a slight tendency toward higher $\Delta h$ for rougher and less steep track segments. Both entrainment and deposition can occur in the transition zone; deposition heights show a large variability with slope angle and large $\Delta h$ can be found both on rough and smooth track segments. In the following we will attempt to simulate this deposition pattern.

\section{Model Equations}

\subsection{Governing Differential Equations}

[17] We describe the mountain profile in a horizontal $X$ and $Y$ coordinate system, given by the metric geographic coordinate system CH1903LN02 provided by the Federal Office of Topography (swisstopo). The elevation of the mountain profile $Z(X, Y)$ is defined for each coordinate pair 

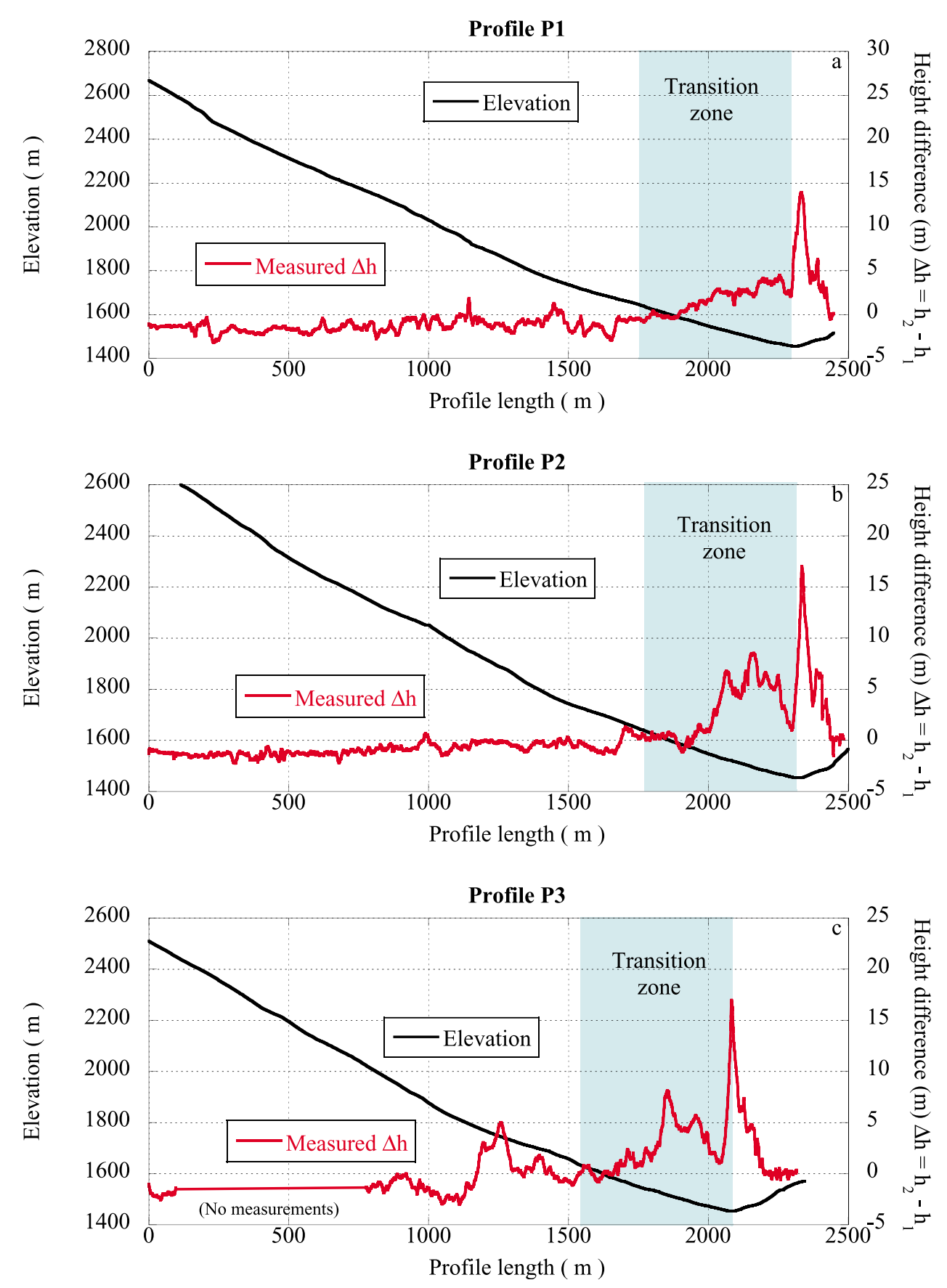

Figure 9. Longitudinal profiles P1-P3. Elevation and measured difference in snow height $\Delta h=h_{2}-h_{1}$. (a) Profile 1, avalanche 817; (b) Profile 2; (c) Profile 3, avalanche 816. Deposition heights vary strongly in the transition zone.

$(X, Y)$. The spacing of the coordinate pairs is typically between $2 \mathrm{~m}$ and $25 \mathrm{~m}$ and has significant impact on the resolution of the simulation domain and results [Maune, 2007; Bühler et al., 2011]. The geographic coordinates are used to construct a local surface-based coordinate system $(x, y, z)$. Using the geographic coordinates, we approximate the surface profile by tangential planes in which the local $z$ direction is defined perpendicular to the plane. The inclination of the planes depends on the resolution of the geographic coordinates. The projection of the local horizontal coordinate directions $x$ and $y$ are defined to be parallel to the geographic coordinates $(X, Y)$. Therefore, the coordinates $x, y$, and $z$ define a surface-induced coordinate system. Its orientation varies with the position on the mountain profile, such that the vector of gravitational acceleration $\boldsymbol{g}=g_{x}, g_{y}, g_{z}$ has in general three nonzero components [Pudasaini and Hutter, 2007; Christen et al., 2010a]. The vector $\boldsymbol{g}=\left(g_{x}, g_{y}, g_{z}\right)$ does not account for small-scale roughness, but larger-scale changes in slope inclination. The accelerations $g_{x}, g_{y}, g_{z}$ do not include centripetal accelerations arising from the intrinsic curvatures of the slope. 

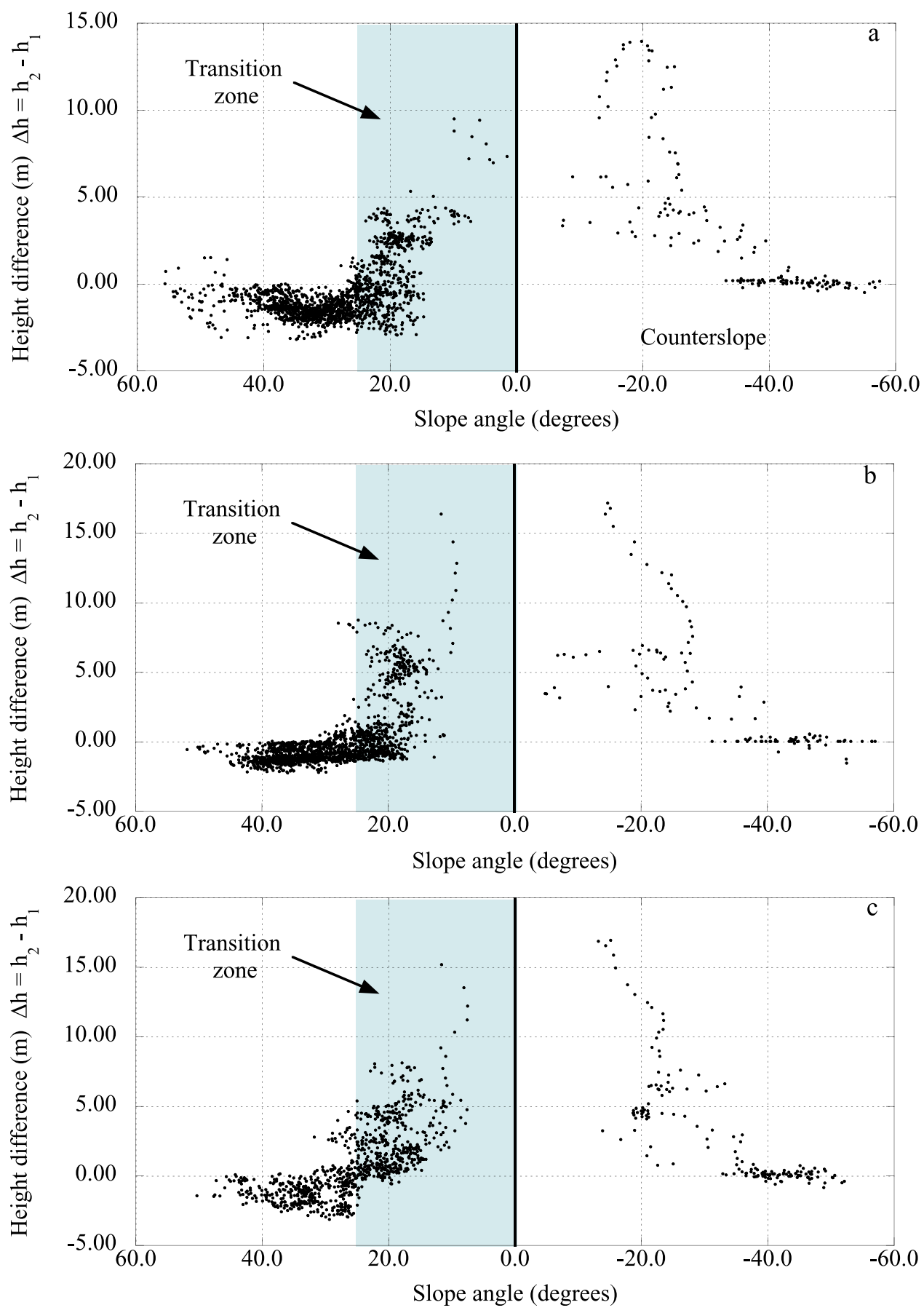

Figure 10. Difference $\Delta h=h_{2}-h_{1}$ as a function of slope angle (summer DEM). (a) Profile P1, (b) Profile P2, and (c) Profile P3. See Figure 4 for profile definition. Large deposition heights are located on the counterslope. Deposition heights vary strongly in the transition zone. Erosion occurs at slope angles greater than $25^{\circ}$.

[18] We solve the following system of depth-averaged partial differential equations in the surface induced coordinate system:

$$
\begin{gathered}
\partial_{t} H+\partial_{x}\left(H U_{x}\right)+\partial_{y}\left(H U_{y}\right)=\dot{Q}(x, y, t), \\
\partial_{t}\left(H U_{x}\right)+\partial_{x}\left(H U_{x}^{2}+g_{z} \frac{H^{2}}{2}\right)+\partial_{y}\left(H U_{x} U_{y}\right)=G_{x}-S_{x},
\end{gathered}
$$

$$
\partial_{t}\left(H U_{y}\right)+\partial_{x}\left(H U_{x} U_{y}\right)+\partial_{y}\left(H U_{y}^{2}+g_{z} \frac{H^{2}}{2}\right)=G_{y}-S_{y},
$$

and

$$
\partial_{t}(H R)+\partial_{x}\left(H R U_{x}\right)+\partial_{y}\left(H R U_{y}\right)=\alpha(\mathrm{S} \cdot \mathrm{U})-\beta(R H) .
$$

The first three equations of this system are the standard depth-averaged mass balance and momentum equations for 

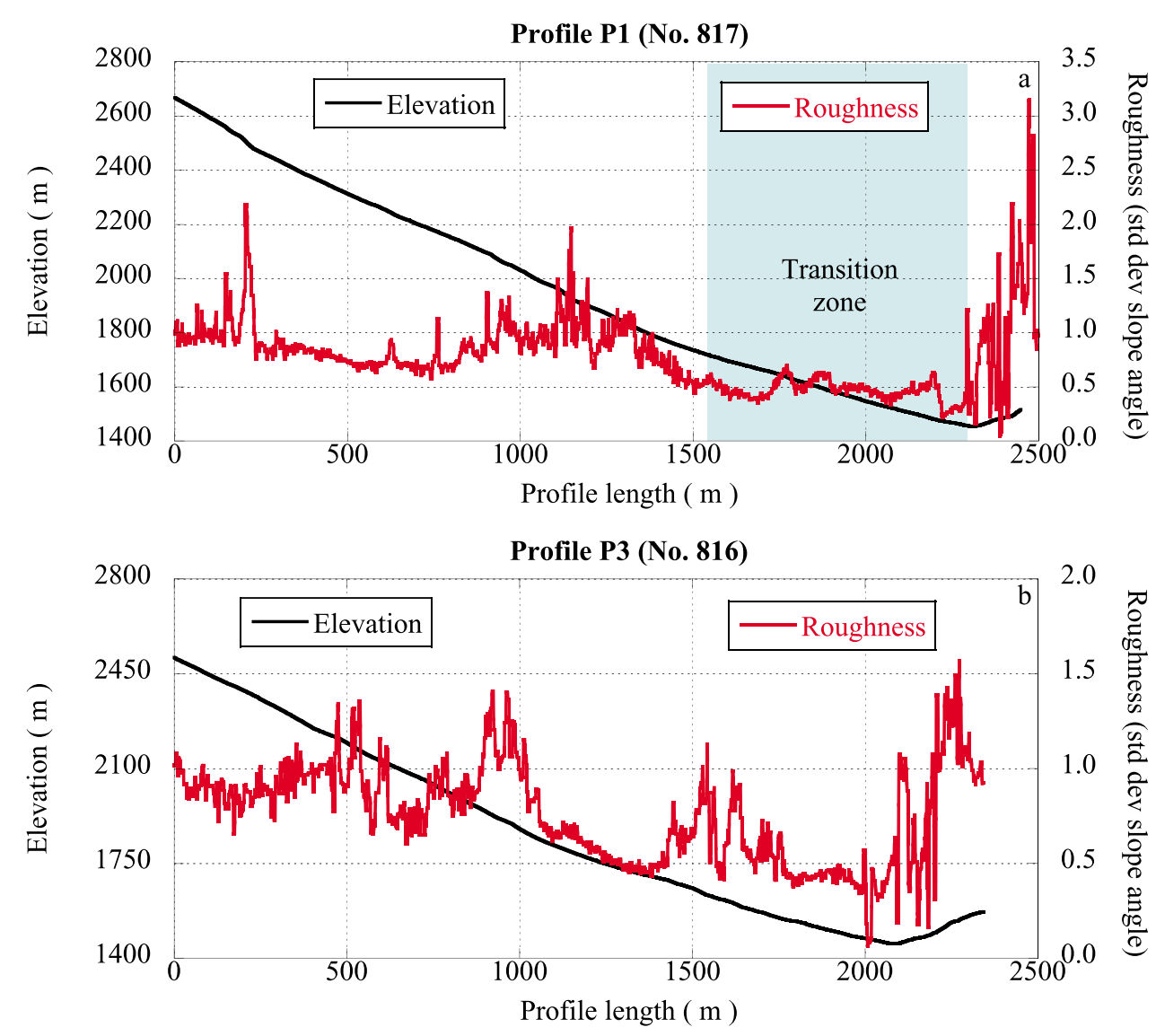

Figure 11. Variation of terrain roughness along longitudinal profiles (a) P1 (avalanche 817) and (b) P3 (avalanche 816).

avalanches [Mangeney et al., 2005; Pudasaini and Hutter, 2007]. The unknown field variables are the avalanche flow height $H(x, y, t)$ and the mean avalanche velocities $U_{x}(x, y, t)$ and $U_{y}(x, y, t)$ in the local $x$ and $y$ directions, respectively. The avalanche flow density $\rho$ is constant. The forth equation, equation (8), is a depth-averaged energy equation accounting for the kinetic energy $R(x, y, t)$ associated with particle velocity fluctuations [Bartelt et al., 2006; Buser and Bartelt, 2009; Christen et al., 2010a]. The field variables are a function of time $t$ and thus we solve the equations from avalanche release $(t=0)$ to avalanche deposition.

[19] The force components associated with the gravitational accelerations in the $x$ and $y$ directions are denoted $G_{x}$ and $G_{y}$ and given by

$$
G_{x}=g_{x} H \quad \text { and } \quad G_{y}=g_{y} H .
$$

Sudden changes in inclination producing additional accelerations are not included in the calculations. Centripetal accelerations can be induced by (1) small-scale surface roughness and (2) mean slope curvature [Pudasaini and Hutter, 2003]. Because real avalanche tracks are smoothed by the snow cover and the avalanches themselves, it is not necessary to include curvature effects from surface roughness. These are included in the friction parameters. Presently our experimental facilities do not allow us to discern the difference between flow rheology and the effects of centripetal forces induced by mean slope curvature. Frictional components $S_{x}$ and $S_{y}$ oppose the gravitational accelerations in the $x$ and $y$ directions. In a depth averaged model, we base our frictional flow laws entirely on mean flow heights and velocities (see section 3.4). In addition we do not modify the hydrostatic pressure terms to include active-passive flow states [Salm, 1966; Savage and Hutter, 1989, 1991; Salm, 1993; Pudasaini and Hutter, 2003; Gray et al., 2003; Pudasaini and Hutter, 2007], as is common in snow avalanche flow calculations [Bartelt et al., 1999].

[20] Mass intake from the snow cover is specified by the volumetric entrainment rate $\dot{Q}(x, y, t)$ per unit area (see section 3.5). The change in momentum $\dot{Q} U_{x}$ and $\dot{Q} U_{y}$ caused by the increase in mass $\dot{Q}$ is considered as an internal mass source. Therefore the momentum change to accelerate the intaken snow is fully accounted for in the total time derivative of the momentum, $\partial_{t}\left(H U_{x}\right)$ and $\partial_{t}\left(H U_{y}\right)$ since $\partial_{t}(H U)=$ $\dot{Q} U+Q \dot{U}$. No external force is necessary to reduce the avalanche velocity (see section 5 examples). Furthermore, we do not consider the momentum change caused by fracturing the snow cover as the fracture energy in new snow is small [Sigrist et al., 2006].

\subsection{Random Kinetic Energy}

[21] Models describing avalanche flow have traditionally neglected the kinetic energy associated with the particle fluctuation velocities, although most authors recognize the importance of the granular effects (see for example Salm [1993] or Perla et al. [1984]). The primary difficulty is introducing a self-consistent energy formulation that divides 

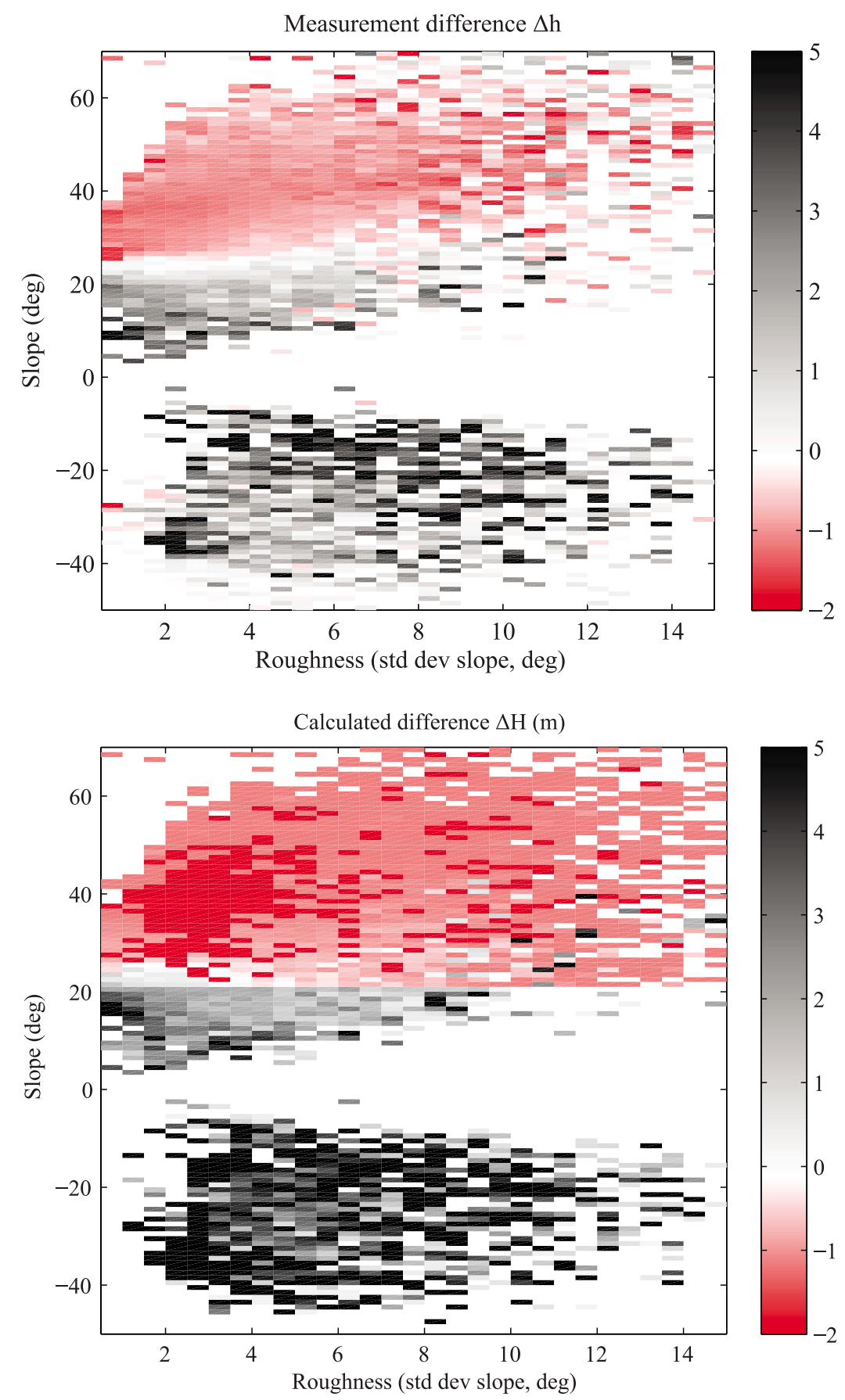

Figure 12. Plots of surface roughness and slope angle for (a) measured height difference $\Delta h$ and (b) calculated height difference $\Delta H$. The plot reveals the influence of surface roughness and slope angle on the depositional behavior of avalanches for a particular avalanche track. In VdlS the depositional behavior is dominated by the counterslope runup. In the transition zone both deposition and entrainment occurs. The height difference $\Delta H$ is found by summing the deposition heights $H^{816}+H^{817}$ from both avalanches. For slope angles above $20^{\circ}$, we subtract the entrainment height $h_{e}=1.15 \mathrm{~m}$ from this value. This leads to a sharp transition between deposition and entrainment at $20^{\circ}$ in the plot. However, in general the model reproduces the observed deposition pattern.

the gravitational work rate into translational kinetic energy and irreversible losses. Because we have some idea of the kinetic energy (we can measure the mean downslope speed of the avalanche), and knowing the drop height (change of potential energy), we can determine the irreversible part.
More specifically, mean values of velocity can be determined by depth-averaging measured velocity profiles in a well defined volume within the avalanche [Kern et al., 2009]. The total avalanche velocity (which we will denote with a hat) is decomposed into varying, mean velocities 


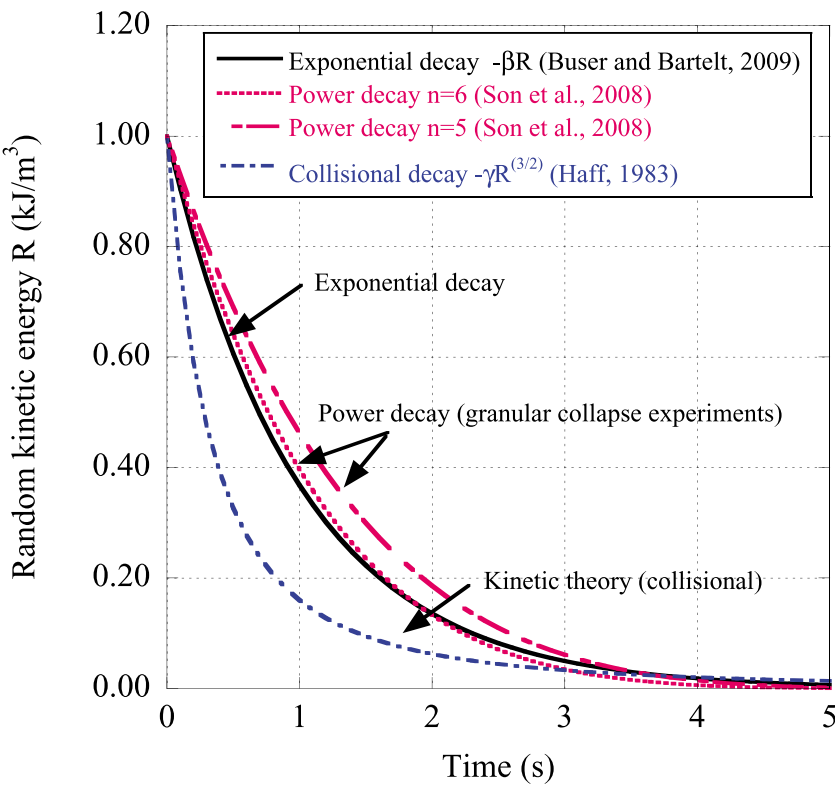

Figure 13. Decay of random kinetic energy in time. Comparison of different decay laws for the case of decay with no production and initial condition $R(t=0)=1 \mathrm{~kJ} / \mathrm{m}^{3}$. Buser and Bartelt [2009] suggest an exponential decay that agrees with the granular collapse experiments in gravity by Son et al. [2008], who fitted decay times with power coefficients varying between $n=3$ and $n=6, R \propto f\left(t^{n}\right)$. The collisional law of Haff [1983] is only valid for collisional systems without enduring particle contacts.

$\left(U_{x}, U_{y}, U_{z}\right)$, and fluctuation velocities $\left(u_{x}, u_{y}, u_{z}\right)$ of the granules in the $x, y$ and $z$ directions, respectively:

$$
\hat{U}_{x}=U_{x}+u_{x} \quad \hat{U}_{y}=U_{y}+u_{y} \quad \hat{U}_{z}=U_{z}+u_{z} .
$$

[22] We assume that the mean velocity in the $z$ direction (slope normal) is $U_{z}=0$. The velocity components not in line with the mean velocity vector are fluctuations and in the mean zero (by definition of the mean). Therefore, we have no slope normal acceleration other than the gravity component $g_{z}$. The kinetic energy $K$ (per unit density) of the reference volume can thus be split into a part associated with the mean translational (slope parallel) motion of the avalanche

$$
K(x, y, t)=\frac{1}{2}\left[U_{x}^{2}+U_{y}^{2}\right],
$$

and a part associated with the fluctuating motion of the granules

$$
\hat{R}(x, y, z, t)=\frac{1}{2}\left[u_{x}^{2}+u_{y}^{2}+u_{z}^{2}\right] .
$$

Because we employ depth-averaged equations, we likewise define the depth-averaged random kinetic energy $R$

$$
R(x, y, t) \equiv \frac{1}{H} \int_{0}^{H} \hat{R}(x, y, z, t) d z .
$$

Any $z$ dependence of $R$ will be lost by the depth-averaging procedure. However, the depth-averaging procedure simplifies the formulation of relationships governing the evolution of $R$ with the model field variables $H$ and $U$.

\subsection{Energy Fluxes}

[23] Although $R$ is mechanical energy, it is internal energy, similar to temperature. As shown in [Buser and Bartelt, 2011] some of this random kinetic energy can be transformed back into potential energy. In this paper we do not consider such effects. The other part of the internal energy is the true thermal energy $E$, produced by the frictional work rate, $\dot{W}_{f}$. The mean frictional work rate $\dot{W}_{f}$ is given by

$$
\dot{W}_{f}=S \cdot U,
$$

the product of the shear stress and mean velocity. The frictional work rate produces thermal energy and random kinetic energy which we split according to the parameter $\alpha$ :

$$
\dot{W}_{f}=\dot{E}+\dot{R}=(1-\alpha) \dot{W}_{f}+\alpha \dot{W}_{f} .
$$

At this stage, the dimensionless coefficient $\alpha$ is introduced only to divide up the total shear work consistently, $\dot{E}=(1-$ $\alpha) \dot{W}_{f}$ and $\dot{R}=\alpha \dot{W}_{f}$. This is simple bookkeeping as we must account for the net change of $R$ in the total energy balance. Therefore, a fraction of the frictional work rate $\alpha \dot{W}_{f}$ is used to produce random energy. The remaining part of the frictional work rate $(1-\alpha) \dot{W}_{f}$ is transformed directly into heat. Because of the eventual and certain decay of $R$ to heat (inelastic interactions), we must add to $\dot{E}$ a part $\beta$ of $R$ that accounts for this irreversible energy flux. The fraction $\beta R$ must be subtracted from the production of $R$. Thus,

$$
\dot{E}=(1-\alpha) \dot{W}_{f}+\beta R \quad \text { and } \quad \dot{R}=\alpha \dot{W}_{f}-\beta R .
$$

This decay $-\beta R$ with constant fraction $\beta$ has been identified in granular collapse experiments under gravity by Son et al. [2008], who fitted the experimental collapse measurements of the decay of $R$ in time with power laws. As can be seen in Figure 13, a power law time decay can be as well approximated by an exponential decay, $\dot{R}=-\beta R$. Kinetic theory models of fluctuation energy decay $\dot{R}=-\gamma R^{3 / 2}$ do not model particles in enduring contact under constant pressure and therefore underpredict decay times [Haff, 1983].

[24] In summary, the derivation of energy equation, equation (8), supplementing the depth-averaged mass and momentum equations follows directly from the irreversibility of the energy associated with random movements and total energy conservation [Bartelt et al., 2006; Pudasaini and Domnik, 2009]. The right-hand side terms of equation (8) represent two additional energy fluxes in avalanches: the first accounting for the production of fluctuation energy (coefficient $\alpha$ ); the second describing its decay (coefficient $\beta$ ). By taking a fraction of the frictional work rate, we make the random kinetic energy $R$ an intermediate energy state between the kinetic energy of the mean flow and the thermal energy, always conserving total energy,

$$
\dot{W}_{g}=\dot{K}+\dot{W}_{f}=\dot{K}+\dot{R}+\dot{E}
$$



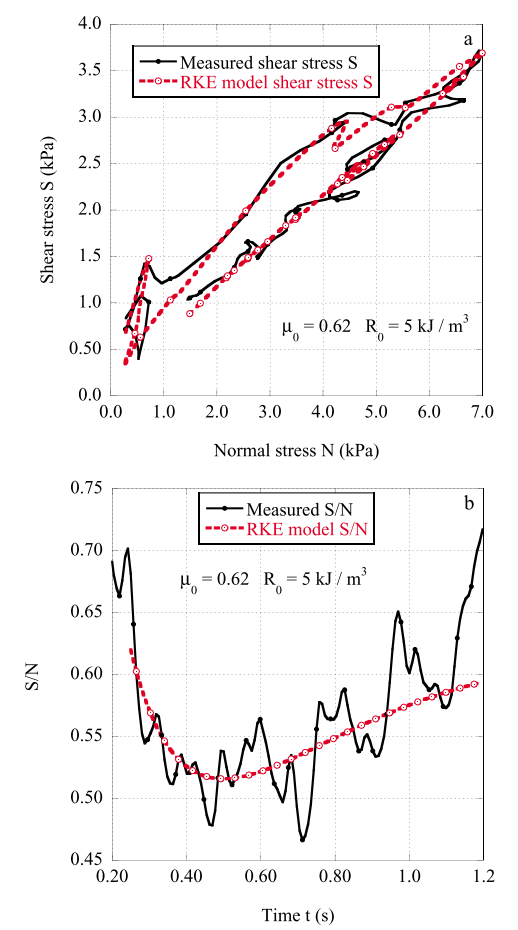

Figure 14. Example results from chute experiments with snow [see Platzer et al., 2007]. (a) Measured shear stress $S$ as a function of measured normal stress $N$; (b) Measured $S / N$ as function of time. Measured results should be compared to idealized curves presented in Figure 15. Note the hysteresis in the measured $S-N$ curve.

where $\dot{W}_{g}$ is the gravitational work rate

$$
\dot{W}_{g}=G_{x} U_{x}+G_{y} U_{y} .
$$

The gravitational work rate is the source of all energy in an avalanche, but it is the frictional work rate that is the source of all inner energy.

\subsection{Extended Voellmy Model $S_{x}$ and $S_{y}$}

[25] Chute experiments have been conducted to measure the basal shear stress $S$ and normal force $N$ of snow [Platzer et al., 2007] and granular [Bartelt et al., 2007; Schaefer et al., 2010] avalanches. In these experiments force plates are positioned at a fixed location on the chute. Flow height sensors measure the avalanche flow height $H$ directly over the plate. Therefore, as the avalanche passes over the plate in time, we have different basal stresses and flow heights as a function of the position within the avalanche. The experiments reveal, for both snow and granular materials, that the ratio $S / N$ differs between the front and tail of the avalanche. The shear stress $S$ is a two-valued function of $N$ (see Figure 14a). This implies that $S / N$ is not a constant, but varies from position to position within the avalanche, which in Figure 14b corresponds to the measurement time. Another result of the experiments is that the stress $N$ equates with the normal component of the weight, indicating no additional accelerations in the $z$ direction.

[26] In full-scale avalanche measurements, the basal stresses cannot be measured because the location of the basal surface is not known. However, the position-dependent behavior is reflected by different velocity profiles [Kern et al., 2009]. The evolution of velocity between the head and tail of the avalanche has been modeled by introducing vertical distributions of random kinetic energy, $R(z, t)$ [Buser and Bartelt, 2009]. Using this procedure, Bagnoldtype velocity profiles at the avalanche head [Norem et al., 1987] and Bingham type profiles at the avalanche tail [Dent et al., 1998] can be modeled with the same material constants $(\alpha$ and $\beta$ ). The different velocity profiles are accounted for by a different amount of random kinetic energy $R$. The current flow state at the given avalanche position can be reproduced only by tracking the history of the random kinetic energy. This result agrees with the basal force plate measurements which show that the depth-averaged values likewise vary with location in the avalanche. This is the primary reason for augmenting the governing differential equations (equations 5-7) with an additional equation describing the net amount of $R$ (equation (8)).

[27] Because the frictional shear stress cannot be determined by the instantaneous state variables (it is two-valued function of $N$ ), we must find a dependency of $S$ on the position-dependent friction coefficients, which become a function of $R$. We use the well-known Voellmy model [Voellmy, 1955; Salm, 1993] by making the Voellmy constitutive parameters $\mu$ and $\xi$ functions of $R$ [Bartelt and Buser, 2010]:

$$
S_{x}=\frac{U_{x}}{\|U\|}\left[\mu(R) g_{z} H+\frac{g\|U\|^{2}}{\xi(R)}\right]
$$

and

$$
S_{y}=\frac{U_{y}}{\|U\|}\left[\mu(R) g_{z} H+\frac{g\|U\|^{2}}{\xi(R)}\right] .
$$

The Voellmy approach splits the total basal friction into a velocity-independent dry Coulomb term which is proportional to the normal stress (friction coefficient $\mu$ ) and a velocity-dependent "viscous" or "turbulent" friction (friction coefficient $\xi$ ) [Salm, 1993]. Interestingly, the approach adopted here is in agreement with the original statements of Voellmy who remarked in his landmark paper, "Die Wirbel der turbulenten Bewegungen verursachen wechselnde Vertikalbeschleunigungen, welche, ähnlich wie bei der Betonvibration, die Wirkung der inneren Reibung und der Zähigkeit herabsetzen, so dass für den weiteren Abfluss weitgehend die Gesetze der Hydraulik gelten." (Translation: The turbulent motion causes fluctuating velocities, which, similar to the vibration of concrete, cause a decrease in the viscosity, such that the usual laws of hydraulics can be employed [Voellmy, 1955, p. 212]).

[28] The force plate measurements show that the amount of $R$ does not increase the pressure $N$. Therefore, because the normal stress does not change (it is in balance with the weight), we must have a change in volume and thus density at the measurement position. This differs from kinetic theory where $R$ increases the pressure in constant volumes [Brilliantov and Pöschel, 2004]. Avalanches have constant pressures with changing volumes because the top surface of the avalanche is a moveable boundary that keeps the pressure constant. When comparing with the theory of granular gases, we have to bear in mind, that in our case we have a 
system with constant pressure, variable volume and, because of mass conversation, of variable density. An increase in random kinetic energy yields a decrease in density, and, as a consequence, a decrease of "inner friction and viscosity," as Voellmy wrote. Therefore we are looking for a function that reduces the Voellmy friction coefficients with increasing $R$. Since the correction must be dimensionless, we have to divide $R$ with $R_{0}$, which we may consider as a scaling factor. The physical interpretation of this factor will later become clear. One of several candidates is the exponential function:

$$
\mu(R)=\mu_{0} \exp \left(-\frac{R}{R_{0}}\right) \quad \text { and } \quad \xi(R)=\xi_{0} \exp \left(\frac{R}{R_{0}}\right) .
$$

The friction coefficients $\mu_{0}$ and $\xi_{0}$ represent the static dry Coulomb and turbulent friction coefficients, respectively

$$
\mu(R=0)=\mu_{0} \quad \text { and } \quad \xi(R=0)=\xi_{0} .
$$

In practice $\mu_{0}$ is the Coulomb friction at release of the avalanche after snow cover collapse. Values between $0.50 \leq \mu_{0}$ $\leq 0.65\left(20^{\circ}\right.$ to $\left.30^{\circ}\right)$ have been measured [Van Herwijnen and Heierli, 2009]. The coefficient $\mu_{0}$ can also be approximated from the angle of repose of the snow deposits at the sides and leading edge of the avalanche. The turbulent friction coefficient can be estimated by studying avalanche tail velocities, assuming that the $\mu_{0}$ is a constant during the descent of the avalanche. We find that $\xi_{0}$ varies between $300 \mathrm{~m} / \mathrm{s}^{2} \leq \xi_{0} \leq 700 \mathrm{~m} / \mathrm{s}^{2}$.

[29] Important is the physical motivation behind the exponential relationship (equation (21)). Random kinetic energy is associated with perpendicular, slope normal movements of the snow granules. The slope normal movements are produced by shearing and cause, in the end, a change in flow density, as any volume must dilate to accommodate the vertical movements. We reduce the flow friction in proportion to the amount of shear, $S$, since the larger the shear $S$ the larger the perpendicular motion of the snow granules and therefore the larger the reduction in density and flow friction. Here the weight of the snow comes into play. The larger the weight, the more $R$ is needed to produce a change in density. The scaling factor $R_{0}$ accounts for this effect and can be approximated by the hydrostatic stress. Measurements in Vallée de la Sionne suggest $5 \mathrm{~kJ} / \mathrm{m}^{3}$ $\leq R_{0} \leq 15 \mathrm{~kJ} / \mathrm{m}^{3}$ [Bartelt and Buser, 2010], which corroborates with measured flow heights between $1 \mathrm{~m}$ and $5 \mathrm{~m}$.

[30] How this formulation describes the measured shear stresses in snow chute experiments is demonstrated in the following example, first for an idealized case (Figure 15) and then with actual measurements from snow chute experiments (Figure 14). For this purpose, we use only the Coulomb part of the Voellmy model. In our idealized case, the avalanche height as a function of time $H(t)$ is known at the measurement location (Figure 15a). The evolution of $R(t)$ is given by equation (8), the competition between production and decay of $R$ (Figure 15b). The random kinetic energy $R(t)$ is maximum near the avalanche front, but diminishes toward the avalanche tail (and therefore in time) as granular interactions dissipate the random kinetic energy produced at the front, where the flow velocity and shear gradients are largest. At the leading edge of the avalanche $H(0)=0$ and $R(0)=0$.
We calculate the shear stress $S$ with the exponential dependence of $\mu$ with $R$. We note that the shear stress $S$ increases and decreases with the flow height $H$, but the $R$ gives rise to the two-valued function with $N$ (Figure $15 \mathrm{c}$ ). We find $S / N$ ratios that vary as a function of location in the avalanche (Figure 15d). This model can now be applied to model snow chute experiments where $S, N$ and $H$ have been measured [Platzer et al., 2007]. Assuming some $R(t)$ we can find frictional constants $\mu_{0}$ and $R_{0}$ which reproduce the measured shear stress and $S / N$ ratios (Figure 14). The constitutive values found from these dry snow experiments, $\mu_{0}=0.62$ and $R_{0}=5 \mathrm{~kJ} / \mathrm{m}^{3}$, will be used in the following to simulate avalanches 816,817 , and 917 .

[31] The standard Voellmy model without memory effects can be recovered from the extended equation system by choosing $\alpha=0$. This always ensures that $R=0$. However, when using the standard Voellmy model $\alpha=0$, the friction constants $\mu_{0}$ and $\xi_{0}$ must be reduced to account for the position-dependent frictional effects.

\subsection{Snow Cover Entrainment}

[32] We define the avalanche release zone to have initial height $H_{0}\left(x_{0}, y_{0}\right)$ where the coordinate pair $\left(x_{0}, y_{0}\right) \in A_{0}$, where $A_{0}$ is the release area. This defines the initial mass of the avalanche.

[33] The quantity $\dot{Q}(x, y, t)$ on the right hand side of the mass balance equation (equation (5)) denotes an additional mass source, referred to as the snow entrainment rate. Thus, the initial mass of the avalanche can increase as a function of $\dot{Q}$. We never extract mass from the flow, therefore $\dot{Q}>0$; no mass escapes the top surface of the avalanche. Avalanche mass is considered deposited when $\mathbf{U}(x, y, t)=0$. This mass still belongs to the avalanche and therefore is not extracted.

[34] The effective entrainment rate $\dot{Q}(x, y, t)$ (that is, the entrainment rate at which the mass is picked up and accelerated) is parameterized by the dimensionless entrainment coefficient $\kappa$. Let $h_{s}(x, y, t)$ represent the total snow cover height which is the sum of different layers $h_{s}=h_{i}$, where $h_{i}$ is the height of the $i$-th layer. The density $\rho_{i}$ of each layer is constant. The snow layers correspond to previous snow fall events or avalanche deposits. The effective entrainment rate is defined according to

$$
\dot{Q}(x, y, t)=\left\{\begin{array}{cc}
\frac{\rho_{i}}{\rho} \kappa_{i} U & \text { for }\left[h_{s}(x, y, 0)-\int_{0}^{t} \dot{Q}(x, y, \tau) d \tau\right]>0 \\
0 & \text { for }\left[h_{s}(x, y, 0)-\int_{0}^{t} \dot{Q}(x, y, \tau) d \tau\right]=0 .
\end{array}\right.
$$

Thus, snow is entrained at the rate $\frac{\rho_{i}}{\rho} \kappa_{i} U$ until no more snow can be entrained. The factor $\frac{\rho_{i}}{\rho}$ scales the entrained mass to the avalanche flow density. Within a single layer, $\kappa_{i}$ is constant. When $\kappa_{i}=1.0$, the entrainment rate is identical to the mean avalanche velocity. However, the snow must be accelerated and therefore a velocity gradient exists at the front of the avalanche. As depth-averaged models do not consider vertical velocity gradients at the avalanche front, the value $\kappa_{i}=1.0$ will overestimate the effective entrainment rate and can therefore be considered an upper bound. Ploughing [Gauer and Issler, 2004] can be modeled with 

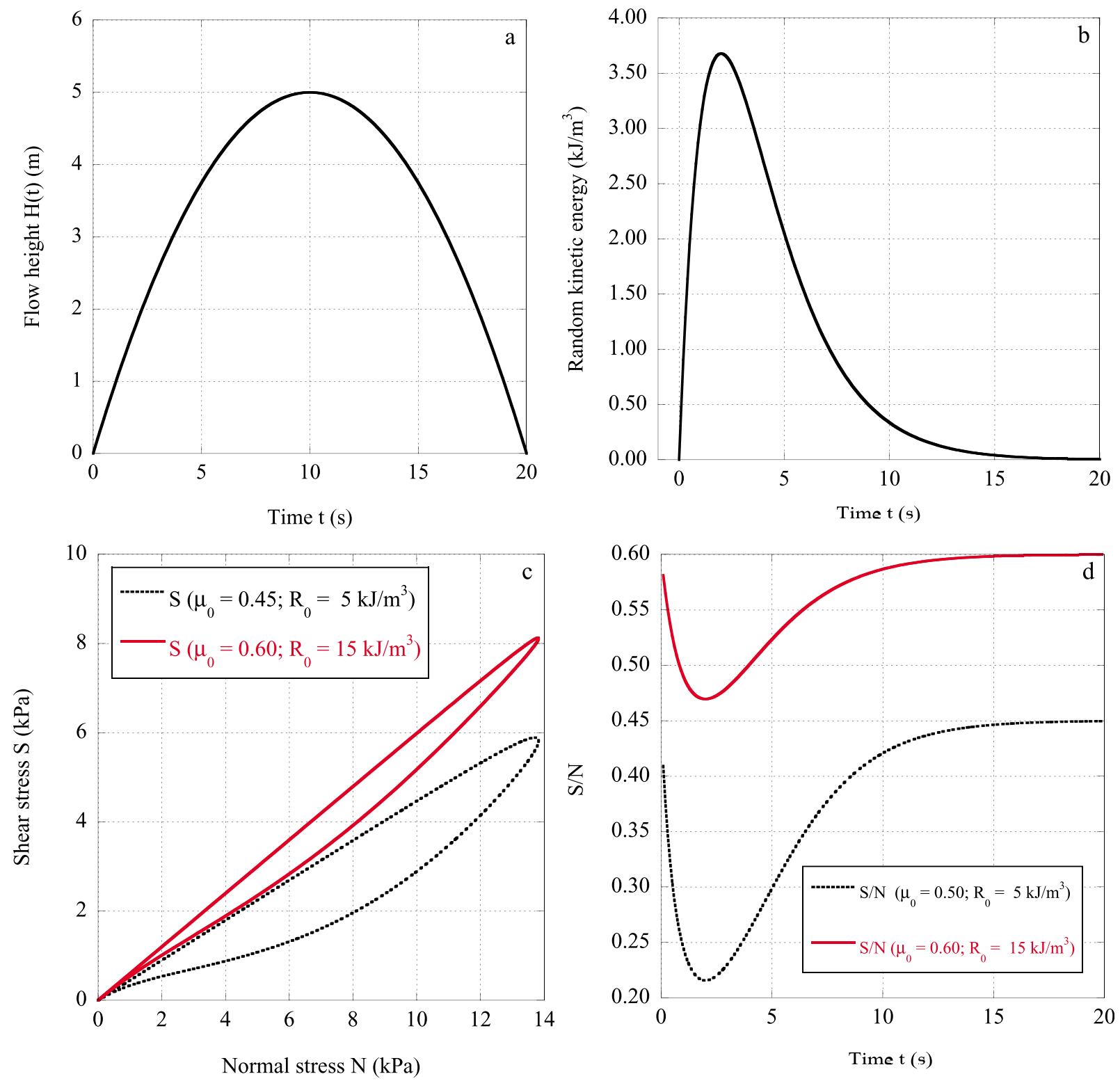

Figure 15. Modelled hysteresis in shear stress. (a) Height input $H(t)$, (b) random kinetic energy input $R(t)$, and (c) calculated shear stress $S$ as a function of $\mu_{0}$ and $R_{0}$. The larger $\mu_{0}$ the steeper the relationship between $S$ and $N$, especially when $R=0$. The smaller $R_{0}$ the larger the shear stress hysteresis.

(d) Calculated $S / N$ ratios. $S / N$ varies with position in the avalanche.

$\kappa_{i} \approx 0.8$ and basal erosion with smaller values of $\kappa_{i}$ [Christen et al., 2010a].

\section{Model Results}

[35] We first simulate avalanche 816 with the standard Voellmy model $(\alpha=0, R=0)$ with and without snow cover entrainment. We apply the Voellmy model in the spirit of a user who has little a posteriori information concerning the measurement details of the avalanche, selecting friction parameters based on avalanche size. We then simulate this avalanche event with the RKE (random kinetic energy) model using production $\alpha$ and decay coefficients $\beta$ derived from velocity profile measurements [Buser and Bartelt, 2009]. We compare the results of these simulations. In both cases we do not vary the model parameters in order to study the simulation results without parameter tuning. The production and decay coefficients of the RKE model are considered material/track constants derived from independent experiments. The deposits predicted by the RKE model are then used to update the summer digital terrain model for the simulations of the second avalanche 817 . Avalanche 817 therefore encounters the deposits (terrain modifications) of avalanche 816 in the runout zone. We then compare the modeled deposition heights of both avalanches $H$ to the measured height difference $\Delta h$. Measured heights are always reported using a small $h$ whereas calculated heights are denoted with large $H$. We will often refer to $\Delta h$ and $H$ as the measured and calculated "deposits," although they are in reality "differences." 

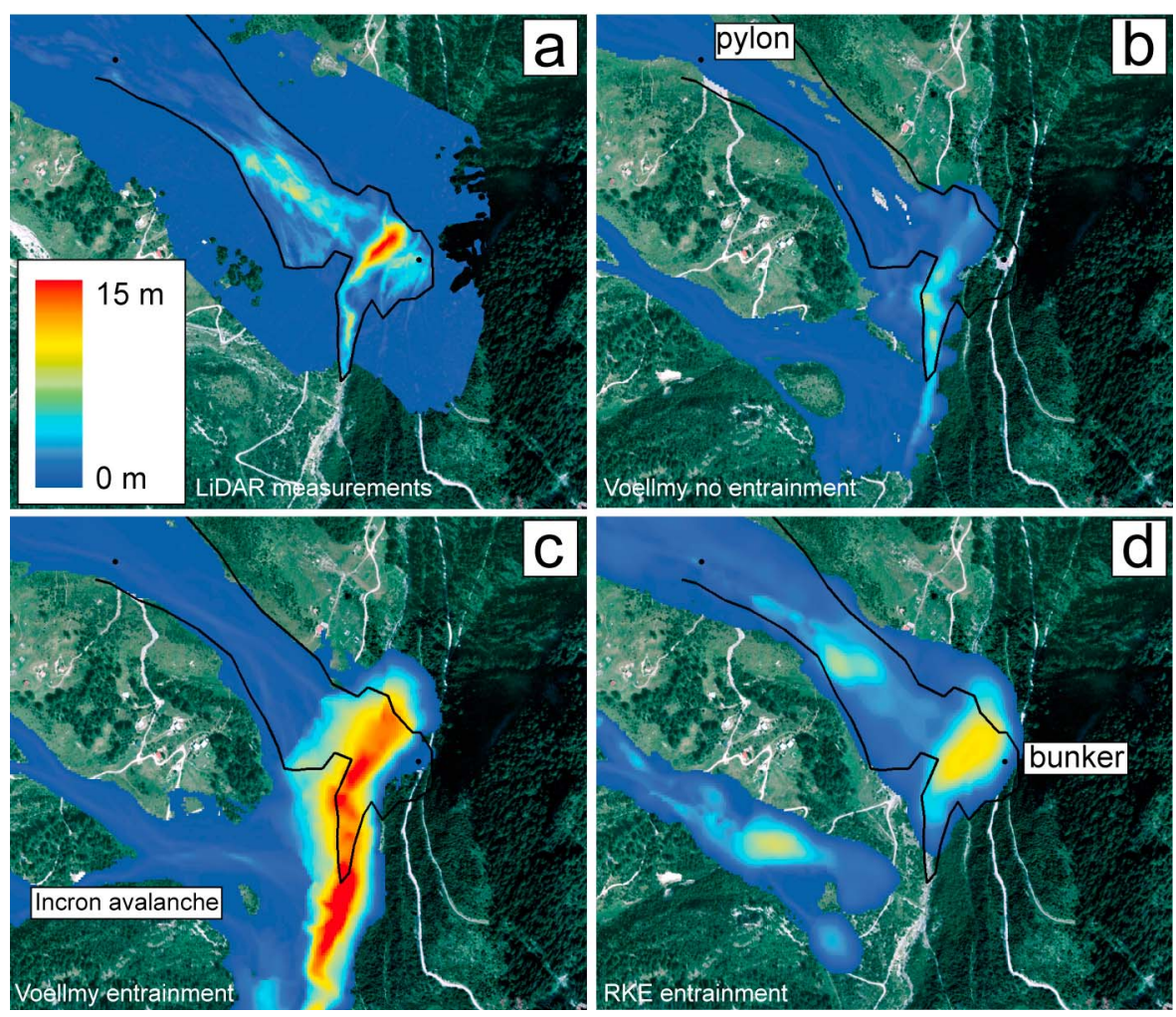

Figure 16. Comparison between measured height difference $\Delta h$ and calculated deposition heights. (a) LiDAR measurements, (b) Voellmy model $(\alpha=0)$, no entrainment, (c) Voellmy model $(\alpha=0)$ with entrainment, and (d) RKE model $(\alpha \neq 0)$ with entrainment.

[36] The slope angle and densification of avalanche deposits must be accounted for when comparing the measurements $h$ to the calculated flow heights $H$. Depthaveraged calculation models assume constant flow density $\rho$ and predict flow heights perpendicular to the slope. Assuming the avalanche deposits have density $\rho_{d}$, the calculated flow heights $H$ are therefore multiplied by the factor $\rho / \rho_{d}$ and divided by the cosine of the slope angle when comparing model calculations to the measurements.

\subsection{Avalanche 816: $\alpha=0, R=0$ (Voellmy)}

[37] We determined the location of the two release zones of the avalanche by tracing postevent aerial photos of the PR and CB1 release areas. Mean fracture depths were determined from the aerial laser scanning measurements, $H_{0}=1.5$ (PR) and $H_{0}=1.0(\mathrm{CB} 1)$. The total release volume $V_{0}$ of this avalanche is so large $V_{0}=103,300 \mathrm{~m}^{3}$ that we can safely assume friction parameters $\mu_{0}$ and $\xi_{0}$ corresponding to an extreme event, $\mu_{0}=0.155$ and $\xi_{0}=2000 \mathrm{~m} / \mathrm{s}^{2}$ [Buser and Frutiger, 1980; Salm et al., 1990; Christen et al., 2010b]. We simulated the avalanche with and without entrainment. For the case with entrainment, we selected $h_{e}=1.15 \mathrm{~m}$ corresponding to the average, measured new snowfall amount. We did not vary the entrainment depth over the track length.

[38] The Voellmy model without entrainment was not able to reproduce the maximum reach of this avalanche (Figure 16); the avalanche ran up the counterslope, but stopped before reaching the bunker. The Voellmy simulations also underestimated maximum avalanche velocity and flow width (Figure 17). No mass is deposited in the transition zone (which does not agree with observations; see Figure 1). The maximum deposition heights on the counterslope are also much smaller than observed (Figures 16a and 16b). The difference between the preavalanche and postavalanche laser scanning measurements is $15 \mathrm{~m}$ in this region; the model predicts only a few meters (Figure 16b).

[39] The standard Voellmy model with entrainment predicts the maximum avalanche velocity as well as the flow width of the avalanche. Deposition heights on the counterslope are too large (the measured $15 \mathrm{~m}$ height arose from both avalanches, not avalanche 816 alone). Even with entrainment, the Voellmy model does not reproduce the measured avalanche deposits in the long transition zone leading up to the counterslope (Figure 18b); the snow deposits are concentrated in the gully and counter slope (Figure 16c).

\subsection{Avalanche 816: $\alpha \neq 0$ (RKE)}

[40] We modeled the avalanche with the RKE model using the same mass inputs as the Voellmy model calculations. The location and volume of the released slabs did not change $\left(V_{0}=103,000 \mathrm{~m}^{3}\right)$; the mass entrainment specifications were not altered $\left(h_{e}=1.15 \mathrm{~m}\right.$ and $\left.\kappa=0.80\right)$.

[41] Our selection for the coefficient $\mu_{0}$ is based on the angle of repose $\phi$ of avalanche deposits, $\phi=32^{\circ}\left(\mu_{0}=\right.$ $\tan \phi=0.62$ ). Such high values of $\mu_{0}$ have been measured at the head and tail of avalanches (Figure 15d) [Platzer et al., 2007]. For $\xi_{0}$ we take low values (with respect to the Swiss guidelines [Salm et al., 1990]) of $\xi_{0}=650 \mathrm{~m} / \mathrm{s}^{2}$. This 


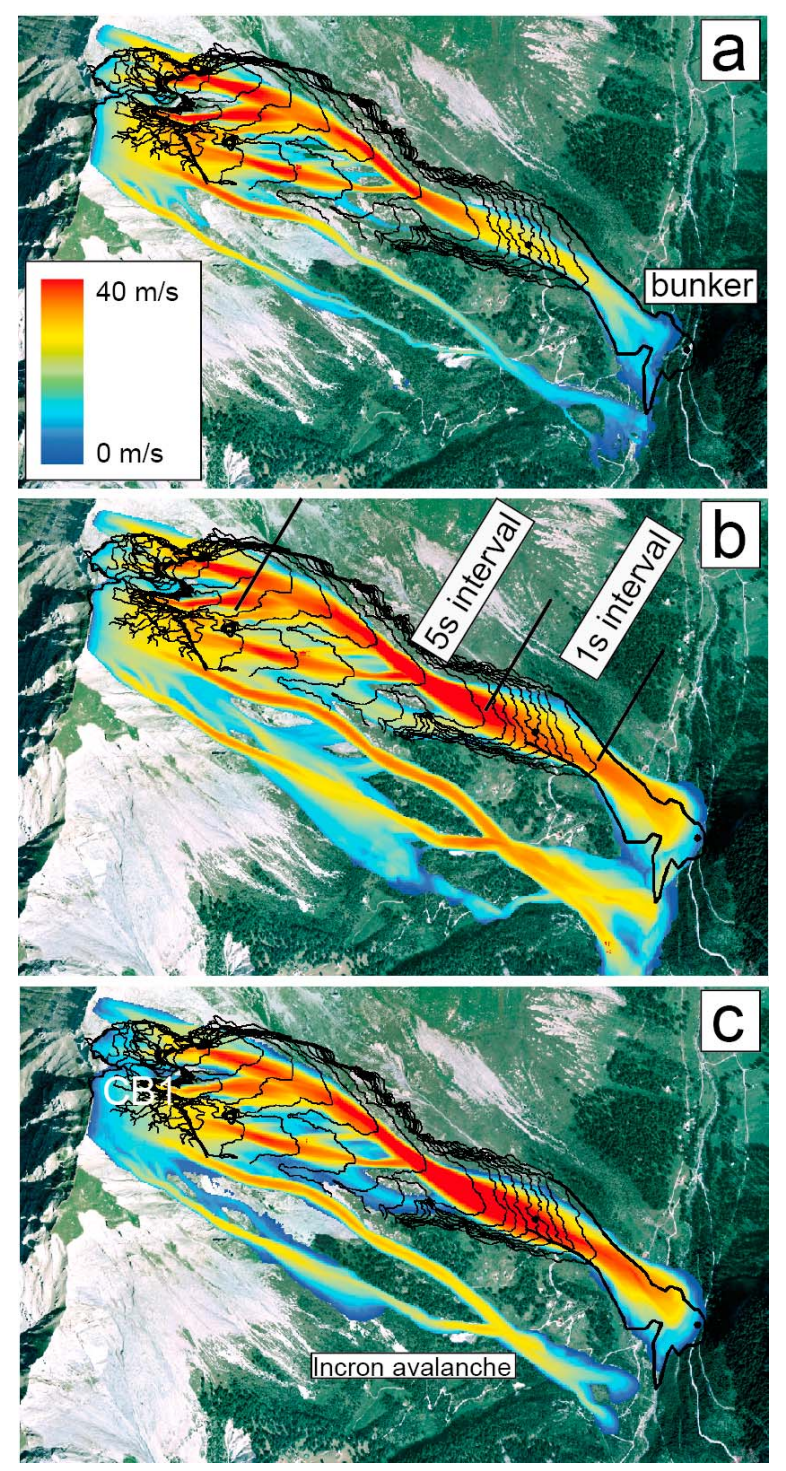

Figure 17. Calculated flow velocities of avalanche 816 . The colors depict the maximum calculated flow velocities. The contour lines trace the location of the avalanche front at known time intervals. (a) Voellmy model $(\alpha=0)$, no entrainment. Avalanche flow widths are too small. (b) Voellmy model $(\alpha=0)$ with entrainment. (c) RKE model $(\alpha \neq 0)$ with entrainment. The avalanche reached maximum velocities of $55 \mathrm{~m} / \mathrm{s}$. Measured leading edge positions are superimposed on the calculations to depict the agreement with the calculated flow direction.

value is close to values originally reported by Voellmy based on back calculations of avalanche damage in Austria [Voellmy, 1955]. When the flow is channelized, this low $\xi_{0}$ value ensures slow, creeping type motion of the avalanche tail.

[42] Measurements of the internal velocity distribution of this avalanche have been reported by Kern et al. [2009]. The highly sheared profiles measured at the front of the avalanche and the plug-type profiles were fitted by Buser and Bartelt [2009] to find the random kinetic energy production $\alpha$ and decay coefficients $\beta$. The ratio $\beta /(1-\alpha)$ balances the dissipative thermal heat fluxes and random kinetic energy fluxes such that a steady flow is possible when the net production of random energy is zero, $\dot{R}=0$ [see Bartelt et al., 2007; Buser and Bartelt, 2009]. This is consistent with the thermodynamic constraint enforcing minimal entropy production in steady state flow [Glansdorff and Prigogine, 1977]. For the measured velocity profiles, Buser and Bartelt [2009] found $0.72 \mathrm{~s}^{-1} \leq \beta /(1-\alpha) \leq 0.88 \mathrm{~s}^{-1}$. To model avalanche 816 (as well as 817), we select $\alpha=0.1$ and $\beta=0.65 \mathrm{~s}^{-1}$; that is, $\beta /(1-\alpha)=0.72 \mathrm{~s}^{-1}$. We select $R_{0}=$ $5 \mathrm{~kJ} / \mathrm{m}^{3}$ based on measurements of other VdlS avalanches [Bartelt and Buser, 2010].

[43] The deposits predicted by the RKE model (Figure 16d) differed strongly from the standard Voellmy model (Figures 16a, 16b, and 17). The RKE model predicts significant deposits in front of the counterslope, in agreement with the laser measurements (compare Figure 18a with $18 \mathrm{~d})$. The avalanche entrained an additional $570,000 \mathrm{~m}^{3}$ of volume; much of this mass was located in the Incron channel and was not part of the deposition region measured by the aerial laser scanning (Figure 18). The total avalanche volume exceeded $670,000 \mathrm{~m}^{3}$. The calculated maximum avalanche velocities (Figure 17c) are near $50 \mathrm{~m} / \mathrm{s}$ and in agreement with the leading edge measurements (Figure 18c). The quality of the agreement depends on the location of the profile; deviations between the measured and calculated velocities can exist when the profile is not located along the main flow direction of the avalanche.

\subsection{Avalanche 817: $\alpha \neq 0$ (RKE)}

[44] We began modeling this avalanche by updating the VdlS terrain model with the deposits calculated for avalanche 816 . The deposition map had a $4 \times 4 \mathrm{~m}$ resolution. To define the avalanche release zone we superimposed postevent, georeferenced aerial photographs of the CB2 release area on the terrain model and traced a closed polygon around the fracture scar. The average release height of the release zone was determined from the aerial laser scanning measurements, $H_{0}=1.3 \mathrm{~m}$. The release mass departed the fracture zone as a single slab with an estimated volume of $V_{0}=$ $119,000 \mathrm{~m}^{3}$.

[45] In the region where the avalanche ran over the deposits of avalanche 816 (transition zone and counterslope) we specified no mass entrainment. In the acceleration zone between $2400 \mathrm{~m}$ and $1900 \mathrm{~m}$ we defined an entrainment height $h_{e}=1.15 \mathrm{~m}$. This height is the mean entrainment depth found from the laser scanning measurements (Figure 4). The entrainment area included the secondary avalanche tracks in the Tsarmettes region. We selected the same entrainment coefficient for the calculations as avalanche $816, \kappa=0.8$. We also did not vary the friction parameters, selecting the same coefficients as avalanche 816: $\mu_{0}=0.62, \xi_{0}=650 \mathrm{~m} / \mathrm{s}^{2}$, $\alpha=0.1$ and $\beta=0.65 \mathrm{~s}^{-1}$. We reasoned that the snow properties did not change significantly in the 50 minute delay between the avalanches.

[46] In the region where the position of the leading edge could be georeferenced, the measured and calculated velocities could be compared on profile $\mathrm{P} 1$. The maximum calculated velocities reached about $55 \mathrm{~m} / \mathrm{s}$ (Figure 19), in good agreement with the observations. In addition the simulated avalanche passed to the orographic left of the pylon, also in 

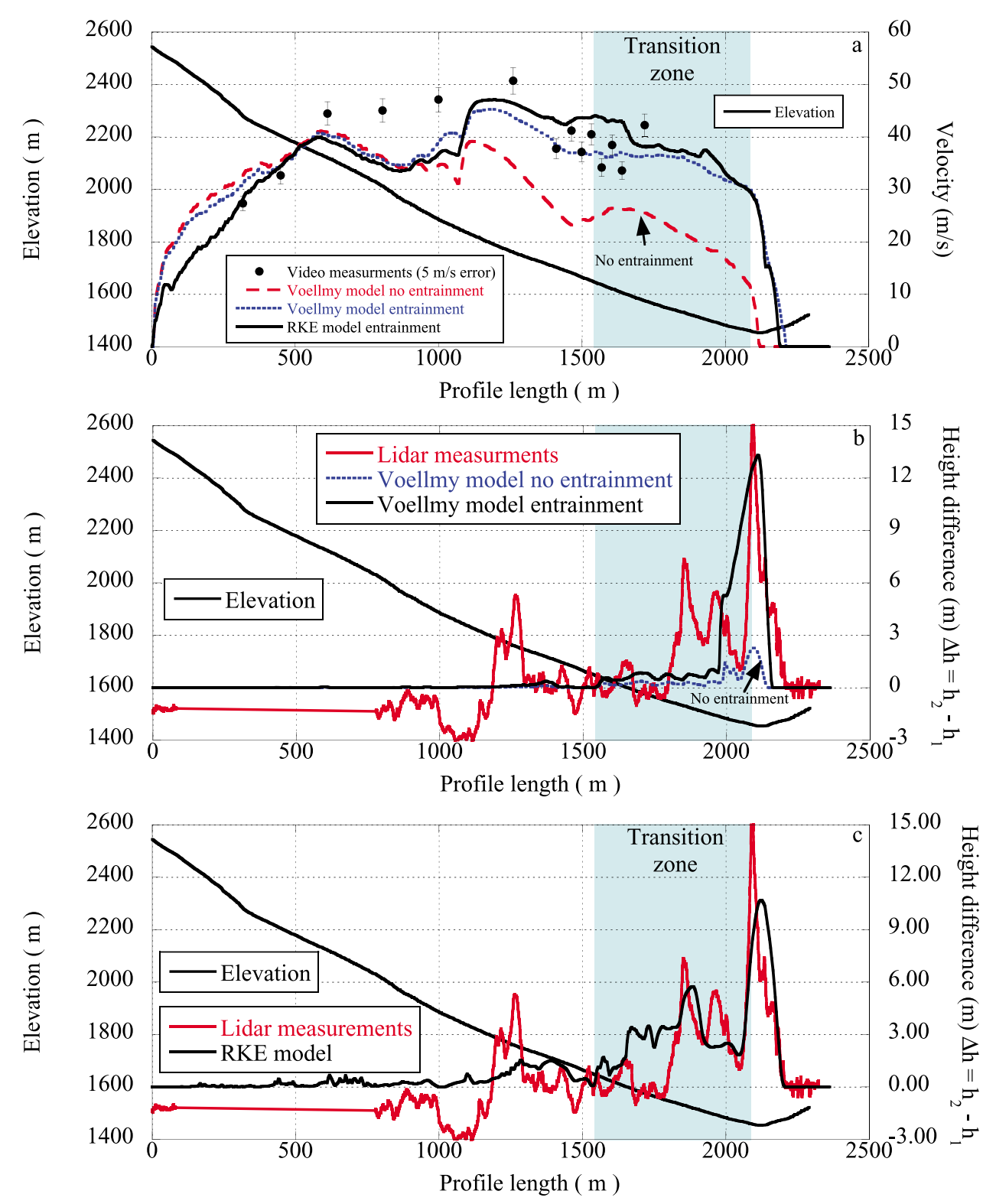

Figure 18. Simulation results of avalanche 816. (a) Comparison between measured and simulated avalanche velocities. Both the Voellmy and RKE models with entrainment simulate the measured velocity in the transition zone. The discrepancy between measured and calculated velocities in the acceleration zone is due to profile alignment. Similar velocities are reached if the profile is shifted sideways. (b) Comparison between measured height difference $\Delta h$ and calculated deposition height using the Voellmy model with and without entrainment. The simulation without entrainment severely underestimates deposition heights; the simulation with entrainment overestimates deposition heights. (c) Comparison between measured $\Delta h$ and calculated deposition height $H$ of the RKE model.

good agreement with the observations. In the acceleration zone the calculated flow width is smaller than the width of the observed powder cloud. This indicates that the powder cloud extends beyond the flow width of the flowing avalanche. The model calculations predict that secondary avalanches descended along the Tsarmettes tracks; these avalanches were observed during the experiment and, as the model predicts, did reach the valley bottom.

[47] To compare the measured difference in laser scanning measurements $\Delta h$ with the calculation results we first summed the deposition heights from the calculation of both avalanches, $H_{d}=H_{816}+H_{817}$. We subtracted the entrainment height $h_{e}$ from $H_{d}$ to model the running surface of the avalanche. In the region where the avalanche ran over the deposits of avalanche 816, we assumed $h_{e}=0$ as no snow was entrained in this region. This procedure provides us with an estimate of the before and after difference in height $\Delta h$ :

$$
\Delta H=\frac{\rho}{\rho_{d}}\left(H_{d}-h_{e}\right) .
$$

To account for densification of the avalanche deposits we multiply the difference with the ratio between $\rho$ the 


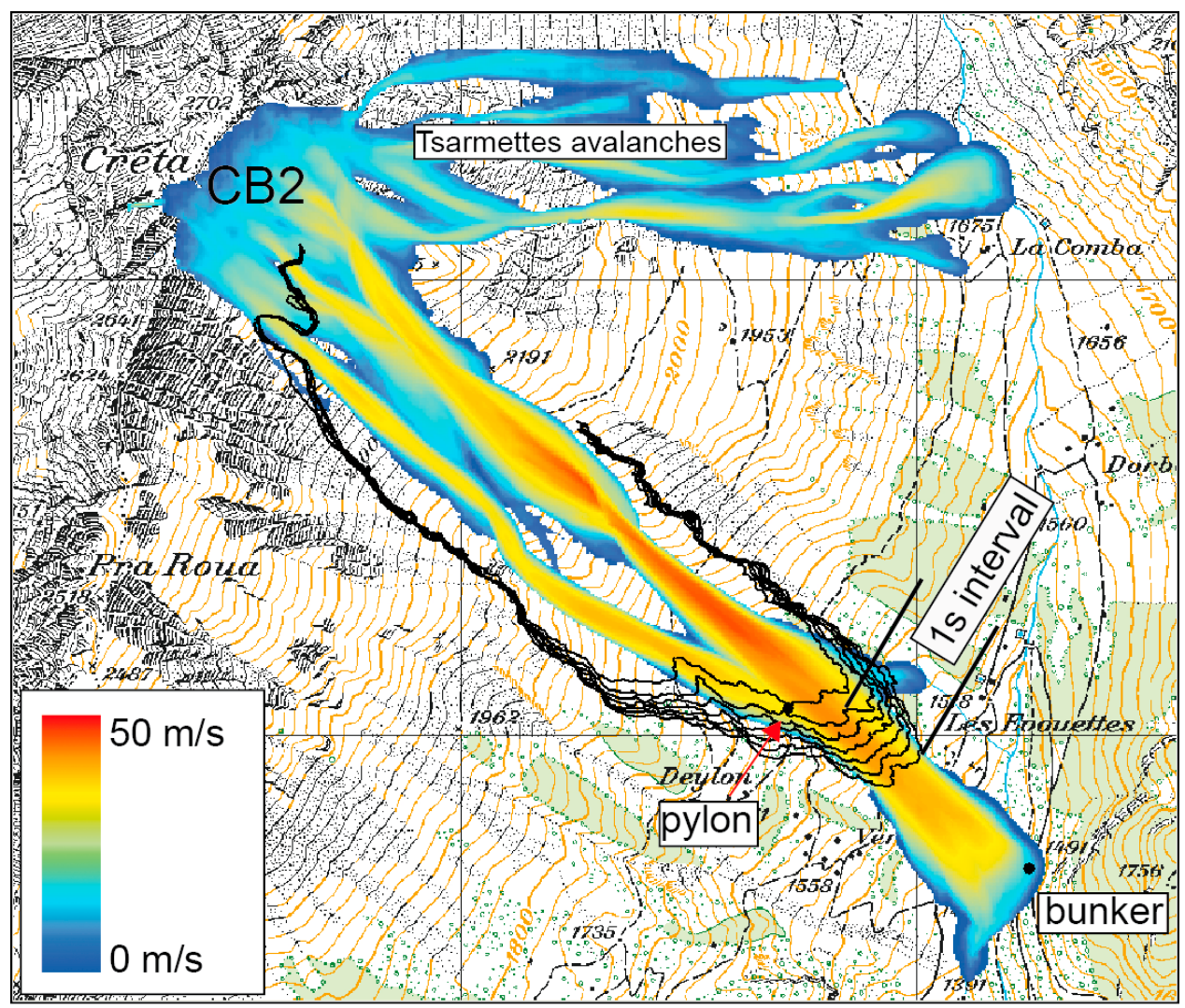

Figure 19. Calculated velocities of avalanche 817 . Peak velocities are on the order of $55 \mathrm{~m} / \mathrm{s}$. The model predicts avalanching in the Tsarmettes region. These avalanches were observed during the experiment. Leading edge profiles of the powder cloud are superimposed. The cloud extends beyond the flow width of the model avalanche.

avalanche density and the deposition density $\rho_{d}$. We took $\rho_{d}=400 \mathrm{~kg} / \mathrm{m}^{3}$. Thus, we compare the calculated deposition heights $\Delta H$ with the measured $\Delta h$ along the three longitudinal profiles P1-P3 (Figure 20) and five lateral profiles P4-P8 (Figure 21).

[48] The comparison reveals that the RKE model captures the main features of the maximum avalanche deposits along the longitudinal profiles (Figure 20) and lateral profile at the La Sionne creek (Figure 21a). The predicted width of the deposits is slightly larger than the observed; the deposition edges have a tendency to diffuse, increasing the width of the deposits and lowering the deposition heights. The calculated deposition peaks are on the order of $12 \mathrm{~m}$. This is in agreement for profile P1, but underestimates the measurements for profiles $\mathrm{P} 2$ and $\mathrm{P} 3$. The nondensified deposits $\left(\rho_{d}=\right.$ $300 \mathrm{~kg} / \mathrm{m}^{3}$, dotted lines in Figure 20) match the observations well, indicating that the choice of deposition density controls how well the measurements and calculations compare.

[49] The avalanche, including the secondary Tsarmettes tracks grew from $V_{0}=119,000 \mathrm{~m}^{3}$ to over $V=635,000 \mathrm{~m}^{3}$. This is slightly smaller than avalanche 816 and is due to our assumption that the first avalanche 816 entrained all the available snow in the transition zone. Avalanche deposits were measured over a $500 \mathrm{~m}$ long region behind the deposition peaks, between profile length $x=1500$ and $x=2300 \mathrm{~m}$ (Figure 20). These deposits are quite high (over $6 \mathrm{~m}, \mathrm{P} 2$ ) and cannot be simulated with the standard Voellmy model. The RKE model is able to model the general extent of the tail deposits, but not some of the detailed inhomogeneous features of the deposits arising from gliding movements or levee formation. This result is apparent in the lateral profiles P4-P8 (Figure 21). At the La Sionne creek (P4, Figure 21a) there is good qualitative and quantitative agreement between the calculations and measurements. The calculated results are smoother than the measurements which lie between the two assumed deposition densities $\rho_{d}=300 \mathrm{~kg} / \mathrm{m}^{3}$ and $\rho_{d}=$ $400 \mathrm{~kg} / \mathrm{m}^{3}$. The avalanche deposits along the lateral profiles P5 and P6 (Figures 21b and 21c) exhibit steep sidewalls: this could not be simulated. The deposition heights $\Delta H$ have the correct order of magnitude and general form of the measured deposition heights $\Delta h$. The uppermost profiles P7 and P8 are more difficult to compare because in these regions snow was unevenly entrained (the running surface of the avalanche is poorly defined); however, the calculated deposition heights $\Delta H$ again have the correct order of magnitude (see Figures $21 \mathrm{~d}$ and $21 \mathrm{e}$ ).

[50] In a final comparison we plotted $\Delta H$ as a function of slope angle and track roughness (Figure 12b). The resulting plot is then compared to the LiDAR measurements $\Delta h$ (Figure 12a). This procedure provides an overall assessment of the simulation, since we are no longer restricted to the profiles, but can consider the entire inundated area. We find a good general agreement; however, the sharp transition between entrainment and deposition in the calculations Figure $12 \mathrm{~b}$ at approximately $20^{\circ}$ is due to our assumption of a constant average entrainment height $h_{e}$. 

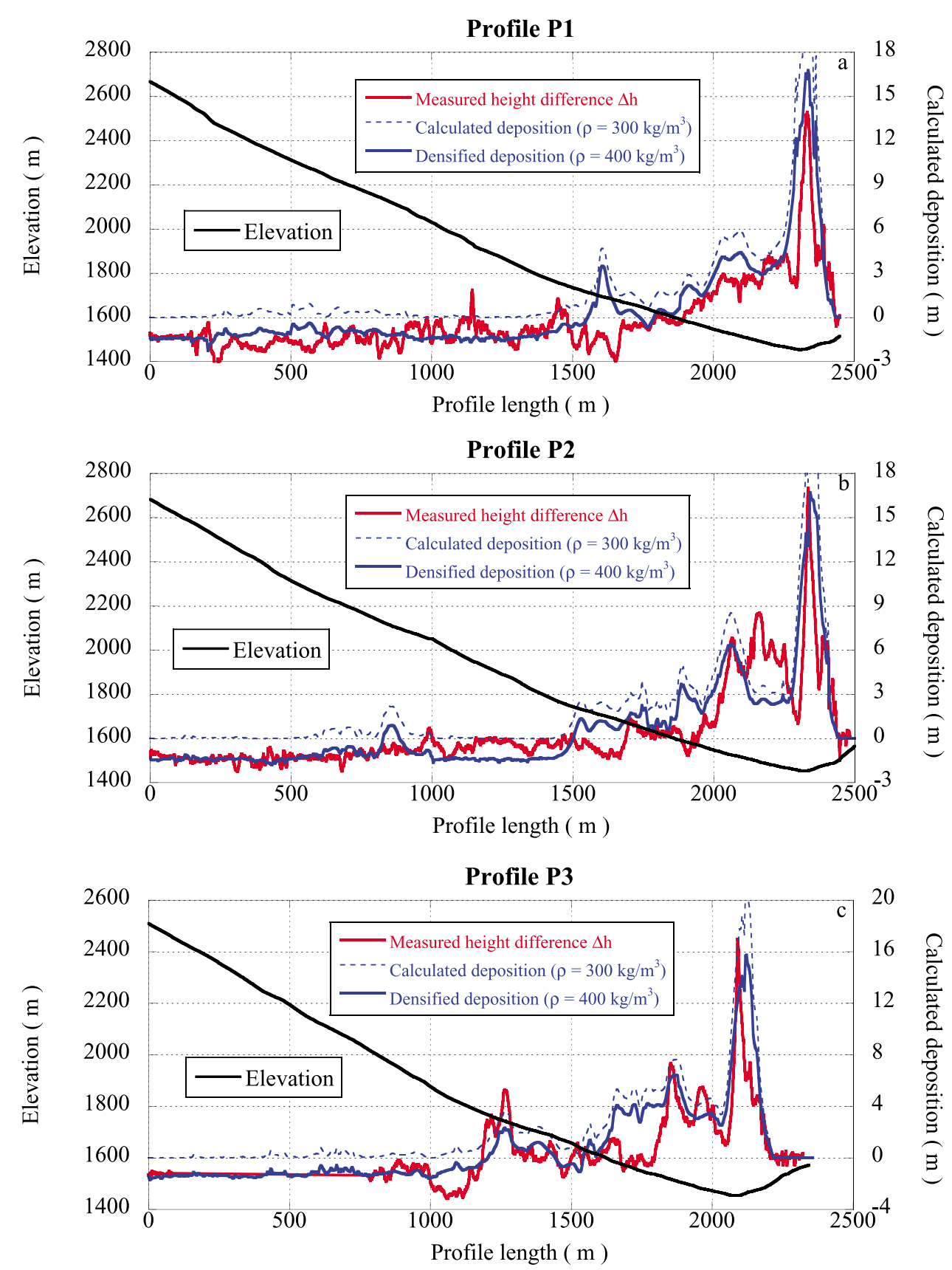

Figure 20. Comparison between LiDAR height difference measurements $\Delta h$ (solid red line) and sum (avalanches 816 and 817) of model calculations along longitudinal profiles (a) P1, (b) P2, and (c) P3. The model calculations were performed using a mean flow density of $\rho=300 \mathrm{~kg} / \mathrm{m}^{3}$ (dotted blue line). These simulation results are depicted without modifications for densification and entrainment. When we account for entrainment and densification $\rho=400 \mathrm{~kg} / \mathrm{m}^{3}$ we obtain the solid blue line. Above profile length coordinate $x=1880 \mathrm{~m}$, the avalanche entrained $h_{e}=1.15 \mathrm{~m}$ of snow.

\subsection{Avalanche 917: $\alpha \neq 0$ (RKE)}

[51] Avalanche 917 was artificially released at 12:45 on 28 March 2008 (Figure 22). The charge released three separate slabs from the PR and CB1 release zones (Figure 23). No laser scanning was performed; however, from video recordings and the postevent photographs we could determine the location of the fracture slabs. One week before the avalanche $0.8 \mathrm{~m}$ of snow was deposited in the release zones.
A bad weather period followed with intermittent snowfall, depositing another $0.2 \mathrm{~m}$ of snow on 24 March. 3 day new snow heights leading up to 28 March were approximately $0.5 \mathrm{~m}$. Therefore, we took the fracture depth of the slabs to be $H_{0}=0.5 \mathrm{~m}$ (release volume $V_{0}=83,000 \mathrm{~m}^{3}$ ). The avalanche descended the orographic right VdlS channel. Over half the released mass entered the secondary Incron channel, especially mass from the PR release area. The frontal lobe of the avalanche flowed to the orographic right hand side of 

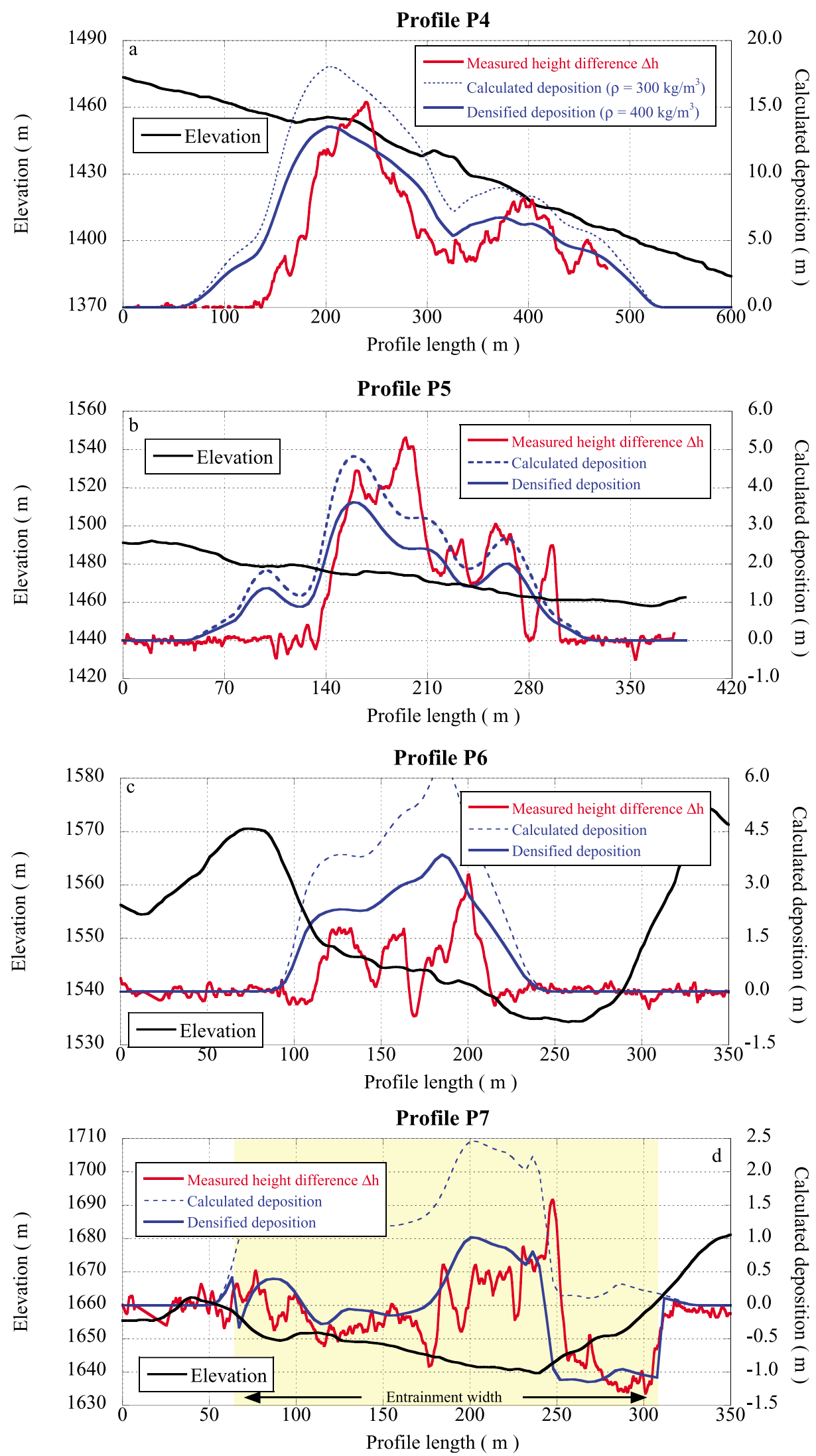

Figure 21. Comparison between LiDAR height difference measurements $\Delta h$ (solid red line) and sum (avalanches 816 and 817) of model calculations along lateral profiles (a) P4, (b) P5, (c) P6, (d) P7, and (e) P8. The model calculations were performed using a mean flow density of $\rho=300 \mathrm{~kg} / \mathrm{m}^{3}$ (dotted blue line). We assume the deposits densify to $\rho=400 \mathrm{~kg} / \mathrm{m}^{3}$ (solid blue line). 


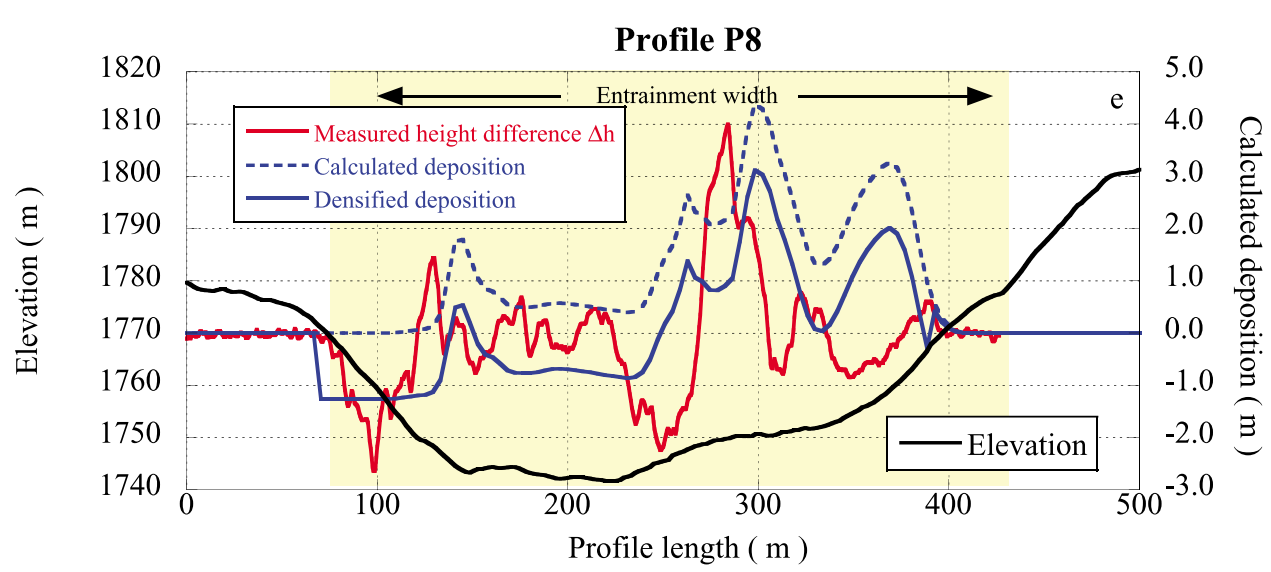

Figure 21. (continued)

the measurement pylon. From the georeferenced video recordings we obtained the speed of the avalanche front between $t=10$ and $t=64 \mathrm{~s}$ (Figure 24). The avalanche reached a terminal velocity of $40 \mathrm{~m} / \mathrm{s}$ in the steep track segments before the transition zone. Since rocky outcrops and vegetation were clearly visible in the region leading up to the counterslope, we estimate the entrainment depth to be small, $h_{e}=0.2 \mathrm{~m}$ (the snowfall of the 24th). The avalanche

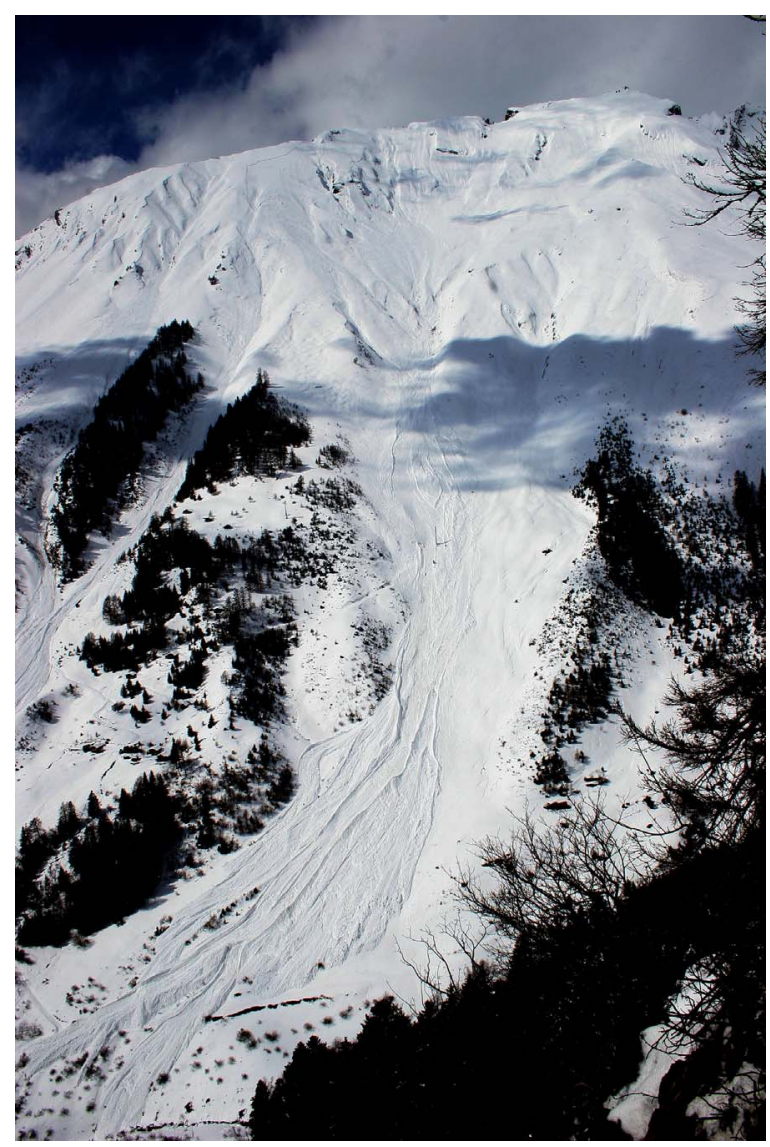

Figure 22. The deposits of avalanche 917 . The avalanche stopped before the counterslope. Flow fingers overran the frontal lobes which stopped before the counterslope. Note the flow channels around the measurement pylon. did not strike the counterslope but stopped in the transition zone before the creek La Sionne (Figure 25). A second avalanche was artificially released from CB2 at 13:00, but no information was gathered from this avalanche.

[52] We calculated avalanche 917 with the same friction parameters as avalanches 816 and $817, \mu_{0}=0.62$ and $\xi_{0}=$ $650 \mathrm{~m} / \mathrm{s}^{2}$. The measured velocity profiles at the tail of the avalanche could be fitted with $\alpha=0.1$ and $\beta=0.63$ (the decay parameter was slightly lower than in avalanche 816; however, we selected $\beta=0.65$ in order to compare simulation results). Because the avalanche entrained little snow, its final volume $\left(V=183,400 \mathrm{~m}^{3}\right)$ was smaller than both avalanches 816 and 817 . This volume includes avalanche mass deposited in the secondary flow channels.

[53] The measured and calculated velocities in the main VdlS track are in good agreement (Figure 24a). Although we do not have actual measurements of deposition heights, from Figure $24 \mathrm{~b}$ we obtain an impression of the deposition pattern. Calculated deposition heights in the transition zone are between 1 and $2 \mathrm{~m}$ (see Figure 22). The RKE model predicts that the avalanche head flows to the orographic right side of the measurement pylon (as observed) and that the avalanche

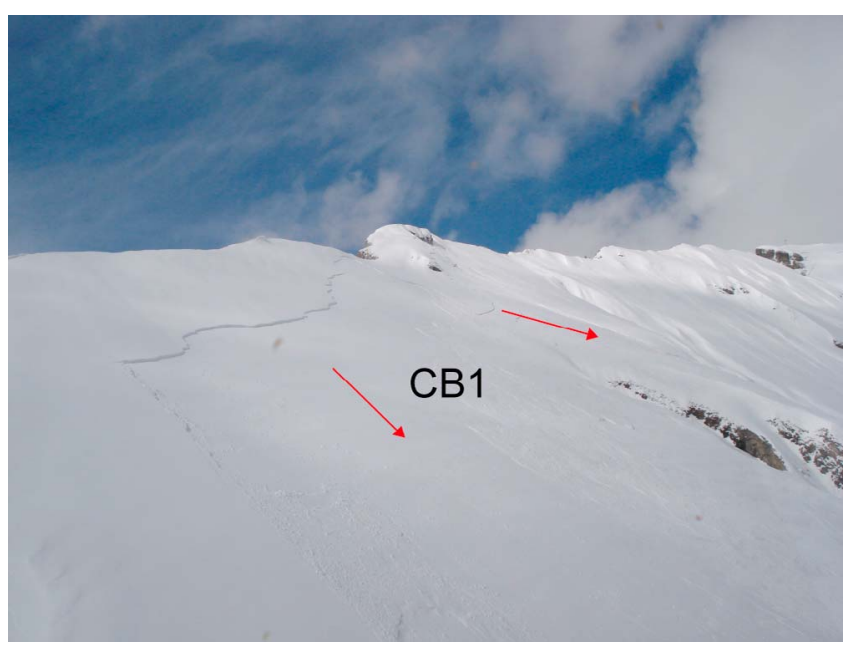

Figure 23. Release zone of avalanche 917, 28 March 2008. Three slabs were artificially released with one charge. The fracture height is approximately $0.5 \mathrm{~m}$. 

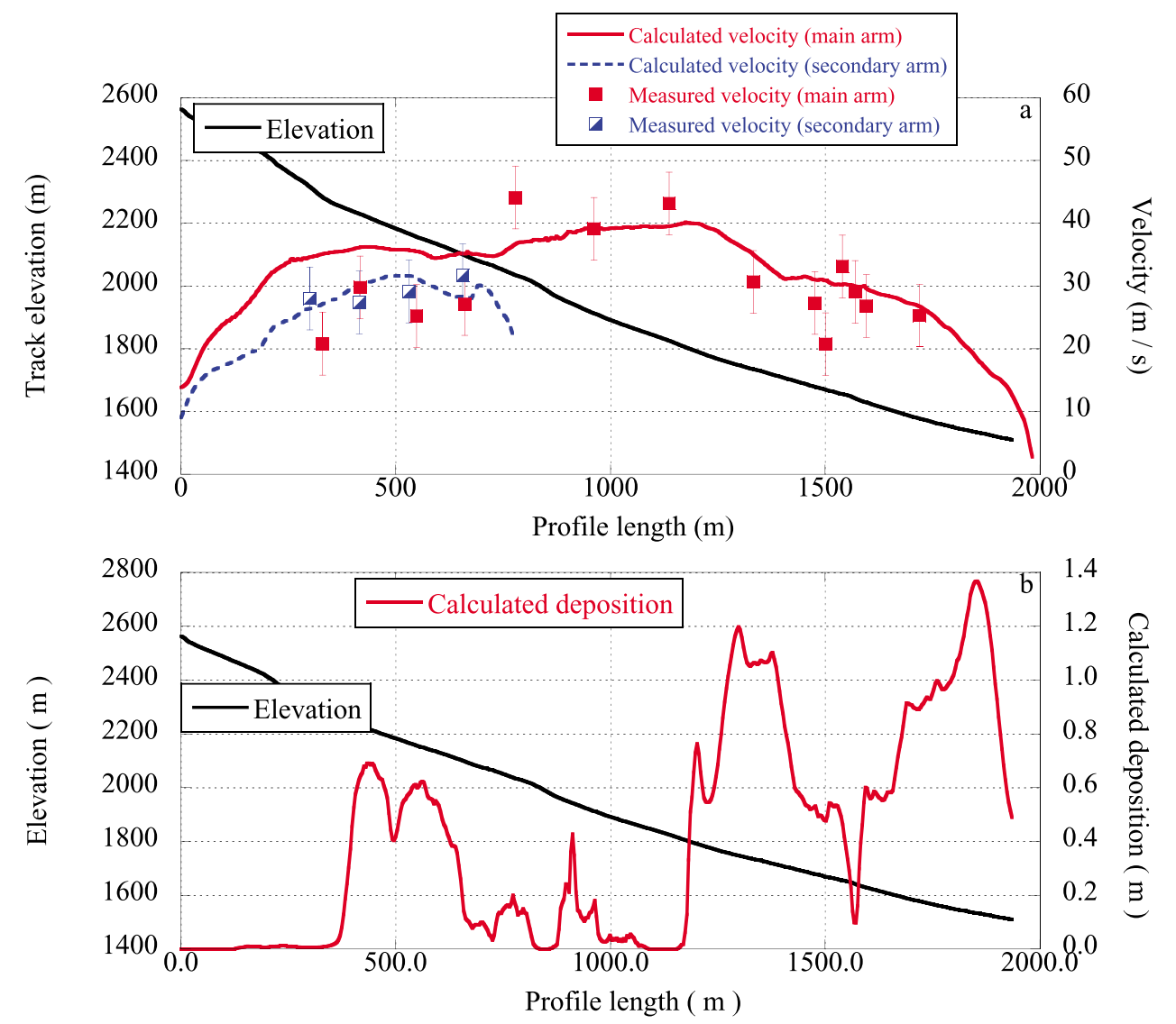

Figure 24. (a) Comparison between the measured and calculated avalanche velocities. Avalanche 917 reached a terminal velocity of $40 \mathrm{~m} / \mathrm{s}$. (b) Calculated deposits. The avalanche ran out on the $20^{\circ}$ transition zone slope. Deposition heights were between 1 and $2 \mathrm{~m}$.

does not reach the La Sionne creek (as observed). The calculated maximum velocities and the measured leading edge velocities are also in good agreement (Figure 25). The maximum simulated velocity at the pylon is $25.4 \mathrm{~m} / \mathrm{s}$ and corresponds well with the optical velocity measurements showing frontal velocities between $22 \mathrm{~m} / \mathrm{s}$ and $26 \mathrm{~m} / \mathrm{s}$ [Kern et al., 2010]. The frontal lobe of the avalanche stops in front of the bunker (as observed) and mass flowing behind the front is slightly shifted to the orographic right side of the transition zone.

\section{Flow Regimes and Snow Entrainment}

[54] To investigate how snow entrainment influences avalanche mobility we return to avalanche 816 . We now simulate avalanche 816 with and without snow cover entrainment using the RKE model. Entrainment calculations have been discussed previously (in sections 4.2 and 4.3). To simulate no entrainment, we set snow cover heights to zero, $h_{s}=0$. The release zone and calculation resolution remained the same.

[55] We first calculated the mean random kinetic energy per unit area $R H$ for both cases (Figure 26). We then laid a track profile through the maximum $R H$ for the case without entrainment and compared avalanche runout distances and velocities (Figure 27). We find that maximum avalanche velocity decreases slightly for the case with entrainment, indicating that energy is required to pickup the snow cover. The reduction in velocity is approximately $5 \mathrm{~m} / \mathrm{s}$. The simulated velocities are within the scatter of the measurements. The calculated flow heights, flow widths, counterslope runup and random kinetic energy densities differ strongly (Figure 26). For the case without entrainment, the avalanche barely reaches the counterslope. The secondary avalanches entering the Incron torrent stop before reaching the valley bottom. Peak random kinetic energy densities are on the order of $20 \mathrm{~kJ} / \mathrm{m}^{2}$ in the runout zone when the avalanche is traveling at some $40 \mathrm{~m} / \mathrm{s}$. This indicates that the mean random kinetic energy is only $4 \%$ of the mean translational kinetic energy density $K$. The calculated fluctuation velocities are on the order of $5 \mathrm{~m} / \mathrm{s}$.

[56] For the case with entrainment (Figure 26b), the random kinetic energy in the runout zone does not decrease as strongly. The production follows surges that are initiated by small deviations in slope angle. These deviations produce maximum mean random kinetic energy densities of $60 \mathrm{~kJ} / \mathrm{m}^{2}$, over $10 \%$ of the total mean translational energy $K$, three times higher than the no entrainment case. As the avalanche impacts the counterslope there is a final explosion of random energy production that is rapidly dissipated as the avalanche runs up the slope. The RKE model predicts that the production of $R$ increases with avalanche mass. This increase in mass not only increases the gravitational work rate $\dot{W}_{g}$, but 

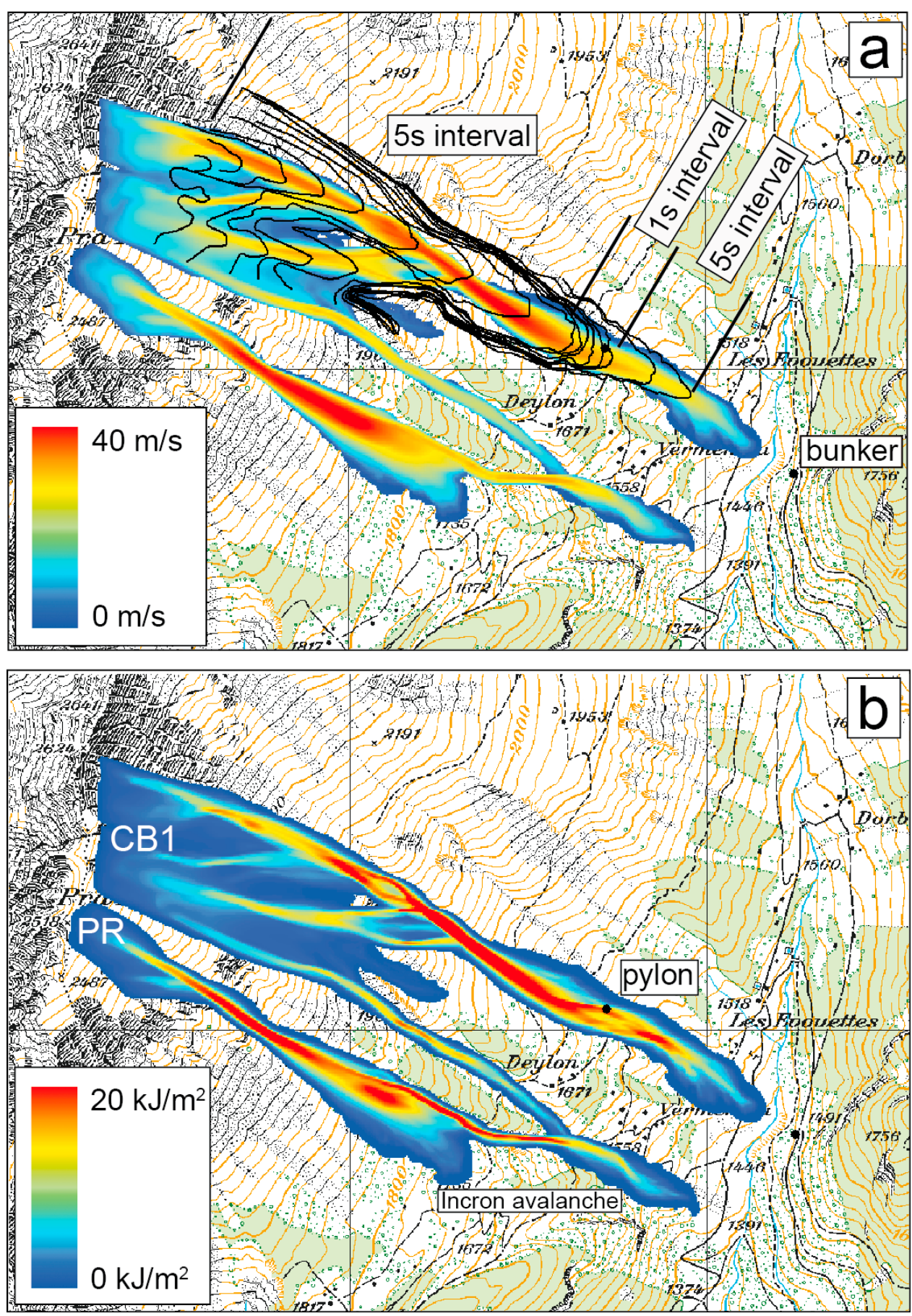

Figure 25. Model calculations of avalanche 917. (a) Maximum calculated avalanche velocities. The avalanche reached velocities of $40 \mathrm{~m} / \mathrm{s}$. Measured leading edge positions are superimposed on the calculations to depict the agreement with the calculated flow direction. (b) Calculated random kinetic energy along the avalanche track.

also the shear stress $S$ and therefore the frictional work rate $\dot{W}_{f}$. This results in an increase of $R$ primarily at the avalanche front where the avalanche velocity and flow heights (because of mass entrainment) are largest. For slope angles above $27^{\circ}$ random kinetic energy increases (Figure 28). The critical angle at which random energy increases or decrease is a function of avalanche mass. Both simulations produce similar runout distances, that are unfortunately controlled by the counterslope at Vallée de la Sionne. However, the total kinetic energy differs. The larger avalanche strikes the counterslope with much higher kinetic energy and would run farther. The simulation without entrainment stops by the time it reaches the creek La Sionne. Another indication of the important role of entrainment is that the secondary avalanches that stop in the Incron torrent are fed with only a 

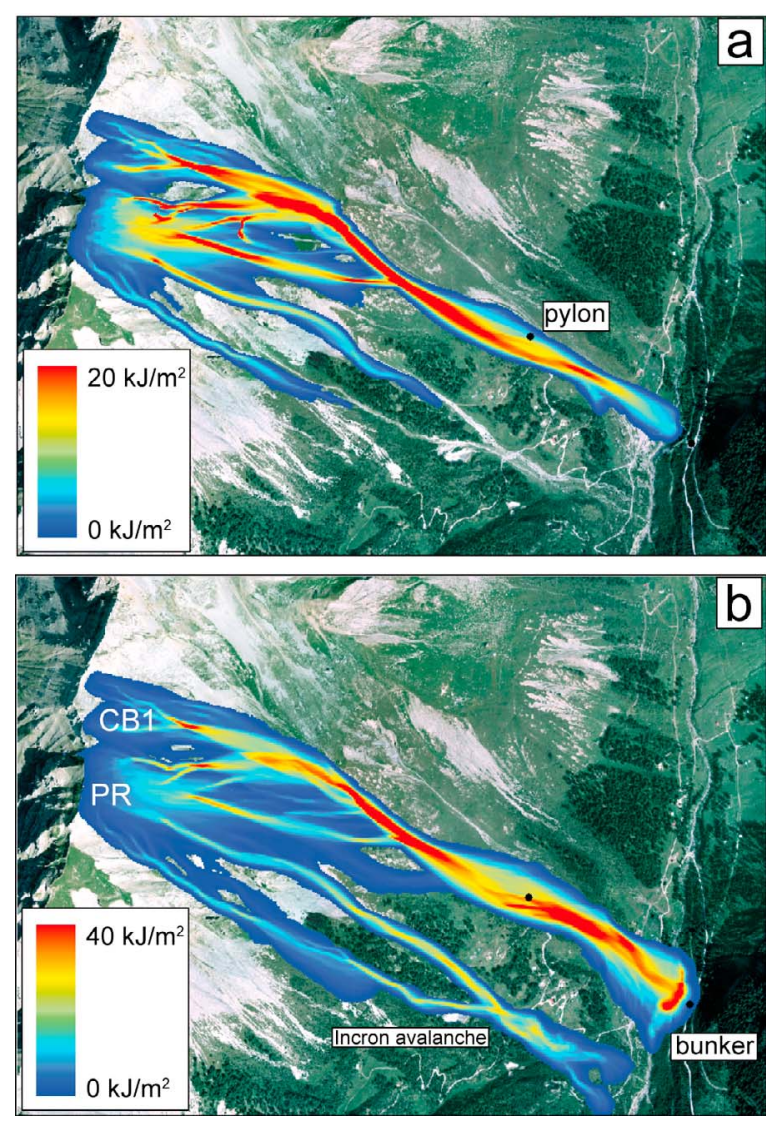

Figure 26. Maximum calculated mean random kinetic energy $R H$ with (a) no entrainment and (b) entrainment. Avalanche velocity and runout of primary avalanche arm are similar; however, flow heights and flow widths differ and counterslope runup differs strongly. Note the difference in runout distance of secondary flow arms. Random energy is generated on the counterslope for the calculation case with entrainment.

small portion of the initial release mass (Figure 26a). Without entraining additional snow, the model predicts that they starve and stop in the steep Incron torrent. This realistic stopping behavior is not modeled by manipulating the friction parameters, but is controlled by the initial mass and snow cover entrainment.

[57] This modeling result is significant, since it provides an explanation for size effects in avalanche flow. Larger avalanches are associated with higher random kinetic energy production and therefore higher fluidization of the avalanche core [Issler and Gauer, 2008; Sovilla et al., 2008], see Figure 27. The fluidization can only be maintained by the mass flux. When the mass flux decreases, at the tail of the avalanche the mass flux always decreases, fluidization ceases and the flow body will form a plug. Motion will stop and we find deposits behind the avalanche front. Of course, considerable basal slip can occur, resulting in slow plug-like fingers that produce a significant shift in deposition, exposing the often observed, smooth ice-like gliding planes in avalanche deposits. This behavior, which is often associated with levee formation, we have not modeled. However, the longitudinal distribution of mass in the avalanche before it reaches the runout zone appears, before it begins to slip, to be governed by the competition between the increase of random energy at the avalanche front and the decrease of random energy at the avalanche tail [Bartelt et al., 2011]. The outcome of this competition, controlled by the mass flux, defines the avalanche flow regime at the front (fluidized, dilute) and the tail (plug).

\section{Conclusions}

[58] The purpose of this paper is to demonstrate how the inclusion of an equation describing the production and decay of the granular fluctuation energy $R$ can be used to describe avalanche motion from initiation to runout. Real-scale snow avalanche measurements were used to validate the model. The speed and fluidization of the avalanche front is controlled by the increase in fluctuation energy, while deposition of avalanche snow is controlled by the decay of the fluctuation energy. We treat friction as a process controlled by the granular energy fluxes. This position-dependent frictional behavior inside the avalanche produces unique deposition patterns depending on mass and terrain. Using the RKE model it is possible to model avalanche deposits on moderate to steep slopes as well as mass effects, such as heightened runout for large avalanches [Legros, 2002]. Flow regime transitions observed at different stages of the avalanche motion can be likewise modeled [Issler and Gauer, 2008; Kogelnig et al., 2011].

[59] We investigated the role of terrain roughness on avalanche flow, defining roughness as the standard deviation from the mean slope angle within a $5 \times 5 \mathrm{~m}$ window using a digital terrain model with a $0.5 \mathrm{~m}$ resolution. The standard deviation varied between only $0.5^{\circ}$ and $1.0^{\circ}$. This result motivated us to simulate the avalanches with a constant production coefficient $\alpha$, which depends mainly on the granular interactions with the running surface. The decay coefficient $\beta$ determines how quickly random kinetic energy is dissipated and is therefore more related to material properties. The distinction between friction parameters that are a function of the material properties and those that are a function of the boundary conditions, might allow us to model avalanches in different terrain with the same material properties. Or, alternatively, avalanches with different material properties on the same track [Christen et al., 2010a].

[60] Smaller-scale terrain features on the order of particle diameters are filled in by the snow cover and leveled out by the avalanche. As the avalanche compacts the snow cover, the "shear roughness," the roughness the avalanche sees, is given by the amount of $R$ within the shear layer, what explains the well known fact that intense snowfall periods in October and November are not associated with far-reaching avalanches, as these avalanches run on bare ground and snow is lost to fill the surface roughness. Large avalanches in high winter see a different terrain and do not loose mass filling up the terrain roughness. Perhaps this is the difference between the arbitrary concept of roughness and slope inclination change: roughness is by definition, filled up by the snow of the avalanche; the change in slope angle induces centripetal accelerations, whereas roughness does not. Because LiDAR based laser scanning is much improving the capability of avalanche engineers to quantify terrain properties [Voesselman and Maas, 2010], it might be possible in 

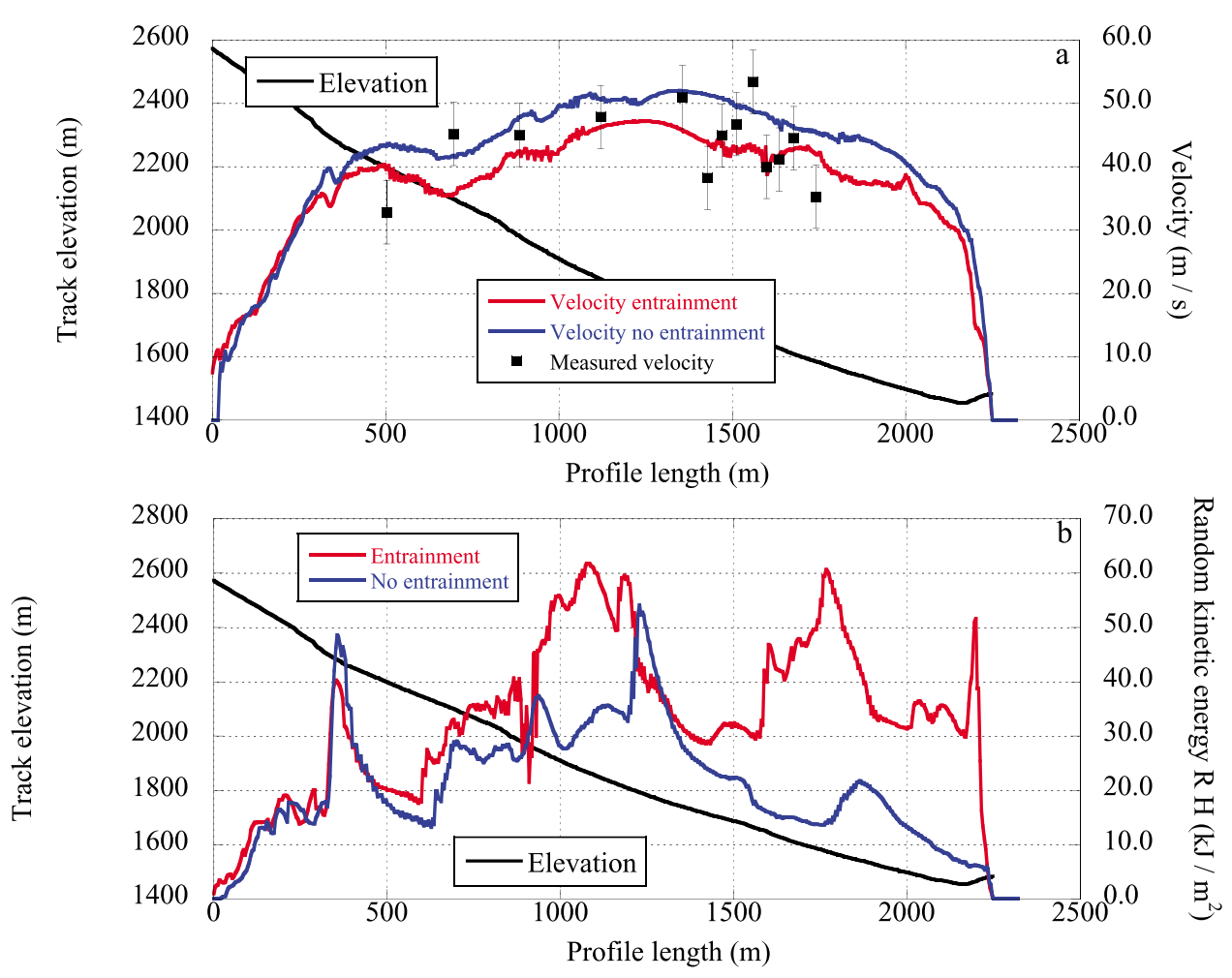

Figure 27. The influence of entrainment on (a) maximum calculated velocity and (b) calculated mean random kinetic energy $R H$ for simulation of avalanche 816 . Mass entrainment strongly influences the production of the random kinetic energy.

the near future to further distinguish between the interaction of material (snow) properties with terrain features. This is a necessary first step to accurately model track sectors where both roughness and centripetal accelerations are influencing the flow.
[61] In the RKE model, gradients of $R$ in the flow direction produce velocity gradients and therefore corresponding height changes (and subsequently hydraulic pressure gradients). This behavior has likewise been modeled by active and passive earth pressure coefficients. The inclusion of both $R$ and earth pressure coefficients is not consistent as

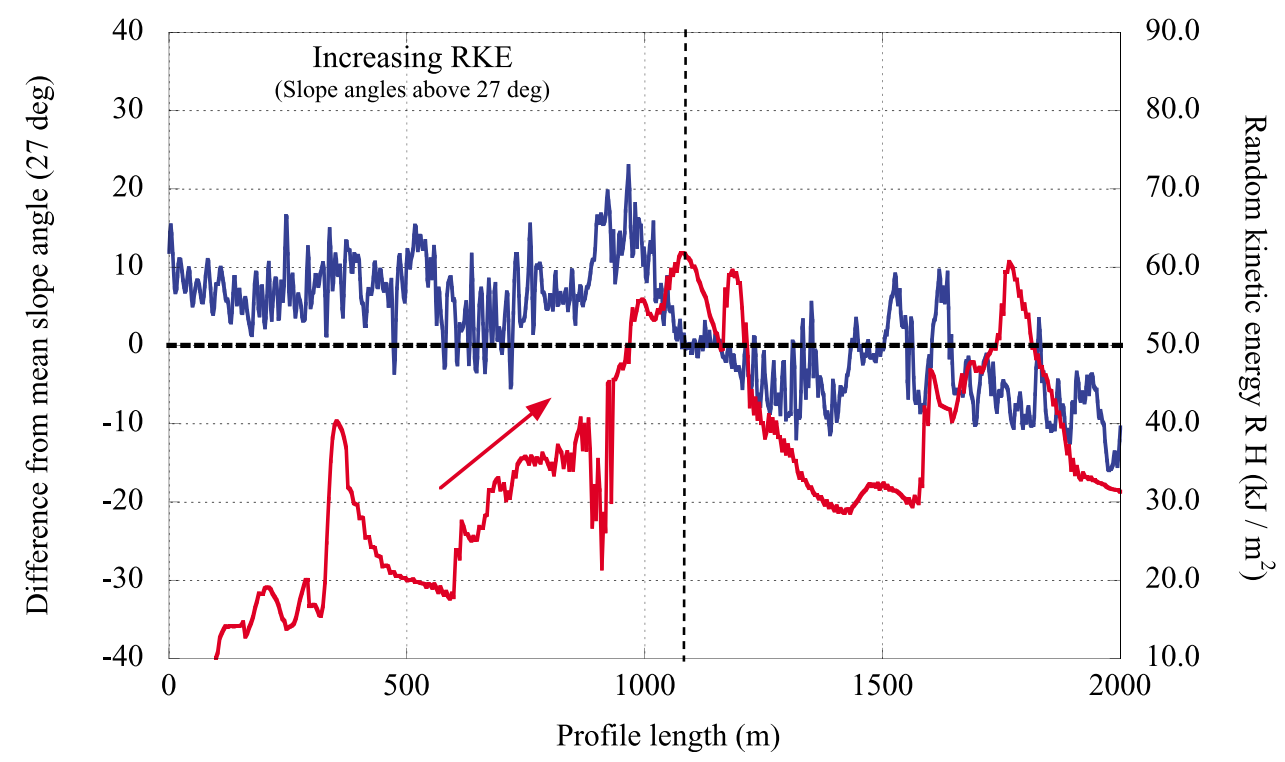

Figure 28. Relationship between slope angle and mean random kinetic energy $R H$ for avalanche 816 . For slope angles above $27^{\circ}$ the mean $R H$ increases. At what angle $R$ increases or decreases depends on avalanche mass. 
each method describes the same phenomena. We have replaced frictional coefficients by a frictional process related to the fluctuation energy $R$. In doing so, we question the need for Earth pressure coefficients and make $\mu_{0}$ and $\xi_{0}$ true constants (mass and slope independent), hopefully replacing the manifold of $(\mu, \xi)$ pairs commonly used in avalanche dynamics calculations. However, for the RKE model to work, it is necessary to accurately specify avalanche release conditions and snow cover entrainment as the results are sensitive to internal mass fluxes. This is difficult to do. For extreme avalanches, considered in avalanche hazard maps, $\mu$ and $\xi$ are known [Buser and Frutiger, 1980; Gruber and Bartelt, 2007] and therefore the RKE model is not really needed.

[62] Acknowledgments. The authors thank Canton Wallis and the head of Natural Hazards, Charly Wuilloud, for their generous and long-lasting support of Vallée de la Sionne. We thank B. Sovilla (SLF, Davos) for the collection of the experimental data. We also thank the technical staff of the SLF for their work in maintaining the experimental infrastructure. Finally, we thank F. Dufour (SLF, Wallis) for his preparation and oversight during the actual experimental observations.

\section{References}

Ancey, C. (2004), Powder snow avalanches: Approximation as nonBoussinesq clouds with a Richardson number-dependent entrainmen function, J. Geophys. Res., 109, F01005, doi:10.1029/2003JF000052.

Bartelt, P., and O. Buser (2010), Frictional relaxation in avalanches, Ann. Glaciol., 51, 98-104.

Bartelt, P., and B. McArdell (2009), Granulometric investigations of snow avalanches, J. Glaciol., 55(193), 829-833.

Bartelt, P., B. Salm, and U. Gruber (1999), Calculating dense-snow avalanche runout using a Voellmy-fluid model with active/passive longitudinal straining, J. Glaciol., 45(150), 242-254.

Bartelt, P., O. Buser, and K. Platzer (2006), Fluctuation-dissipation relations for granular snow avalanches, J. Glaciol., 52(179), 631-643.

Bartelt, P., O. Buser, and K. Platzer (2007), Starving avalanches: Frictional mechanisms at the tails of finite-sized mass movements, Geophys. Res. Lett., 34, L20407 doi:10.1029/2007GL031352.

Bartelt, P., L. Meier, and O. Buser (2011), Snow avalanche flow regime transitions induced by mass and random kinetic energy fluxes, Ann. Glaciol., 58, 159-164.

Brilliantov, N., and T. Pöschel (2004), Kinetic Theory of Granular Gases, 329 pp., Oxford Univ. Press, New York.

Bühler, Y., M. Christen, J. Kowalski, and P. Bartelt (2011), Sensitivity of snow avalanche simulations to digital elevation model quality and resolution, Ann. Glaciol., 58, 72-80.

Buser, O., and P. Bartelt (2009), Production and decay of random kinetic energy in granular snow avalanches, J. Glaciol., 55(189), 3-12.

Buser, O., and P. Bartelt (2011), Dispersive pressure and density variations in granular snow avalanches, J. Glaciol., 57(205), 857-860.

Buser, O., and H. Frutiger (1980), Observed maximum run-out distance of snow avalanches and the determination of the friction coefficients $\mu$ and $\xi$, J. Glaciol., 26(94), 121-130.

Christen, M., J. Kowalski, and P. Bartelt (2010a), RAMMS: Numerical simulation of dense snow avalanches in three-dimensional terrain, Cold Reg. Sci. Technol., 63, 1-14.

Christen, M., P. Bartelt, and J. Kowalski (2010b), Back calculation of the In den Arelen avalanche with RAMMS: Interpretation of model results, Ann. Glaciol., 51, 161-168.

Dent, J. D., K. J. Burrell, D. S. Schmidt, M. Louge, E. E. Adams, and T. G. Jazbutis (1998), Density, velocity, and friction measurements in a dry-snow avalanche, Ann. Glaciol., 26, 247-252.

Fukushima, Y., and G. Parker (1990), Numerical-simulation of powdersnow avalanches, J. Glaciol., 36(123), 229-237.

Gauer, P., and D. Issler (2004), Possible erosion mechanisms in snow avalanches, Ann. Glaciol., 38, 384-392.

Gauer, P., D. Issler, K. Lied, K. Kristensen, H. Iwe, E. Lied, L. Rammer, and H. Schreiber (2007a), On full-scale avalanche measurements at the Ryggfonn test site Norway, Cold Reg. Sci. Technol., 49, 39-53.

Gauer, P., M. Kern, K. Kristensen, K. Lied, L. Rammer, and H. Schreiber (2007b), On pulsed Doppler radar measurements of avalanches and their implication to avalanche dynamics, Cold Reg. Sci. Technol., 50, 55-71.
Gauer, P., K. Lied, and K. Kristensen (2009), Analysis of avalanche measurements out of the runout area of NGI's full-scale test-site Ryggfonn, Cold Reg. Sci. Technol., 57, 1-6.

Glansdorff, P., and I. Prigogine (1977), Thermodynamic Theory of Structure, Stability, and Fluctuations, Wiley-Interscience, London.

Gruber, U., and P. Bartelt (2007), Snow avalanche hazard modelling of large areas using shallow water numerical methods and GIS, Environ. Modell. Software, 22(10), 1472-1481.

Gray, J. M. N. T., Y. Tai, and S. Noelle (2003) Shock waves, dead zones, and particle-free regions in rapid granular free-surface flows, J. Fluid Mech., 491, 161-181

Haff, P. (1983), Grain flow as a fluid-mechanical phenomenon, J. Fluid Mech., 134, 401-430.

Hungr, O. (1995), A model for the runout analysis of rapid flow slides, debris flows, and avalanches, Can. Geotech. J., 32, 610-623.

Issler, D., and P. Gauer (2008), Exploring the significance of the fluidized flow regime for avalanche hazard mapping, Ann. Glaciol., 49, 193-198.

Jomelli, V., and P. Bertran (2001), Wet snow avalanche deposits in the French Alps: Structure and sedimentology, Geogr. Ann., Ser. A, 83(1-2), 15-28.

Kern, M., P. Bartelt, B. Sovilla, and O. Buser (2009), Measured shear rates in large dry and wet snow avalanches, J. Glaciol., 55(190), 327-338.

Kern, M., P. Bartelt, and B. Sovilla (2010), Velocity profile inversion in dense avalanche flow, Ann. Glaciol., 51, 27-31.

Kogelnig, A., E. Suriñach, I. Vilajosana, J. Hübl, B. Sovilla, M. Hiller, and F. Dufour (2011), On the complementariness of infrasound and seismic sensors for monitoring snow avalanches, Nat. Hazards Earth Syst. Sci. 11(8), 2355-2370.

Legros, F. (2002), The mobility of long-runout landslides, Eng. Geol., 63(3-4), 301-331.

Mangeney-Castelnau, A., F. Bouchut, J. P. Vilotte, E. Lajeunesse, A. Aubertin, and M. Pirulli (2005), On the use of Saint Venant equations to simulate the spreading of a granular mass, J. Geophys. Res., 110, B09103, doi:10.1029/2004JB003161.

Maune, D. (2007), Digital Elevation Model Technologies and Applications: The DEM User Manual, 655 pp., Am. Soc. for Photogramm. and Remote Sens., Bethesda, Md.

Naaim, M., F. Naaim-Bouvet, and T. Faug (2003), Dry granular flow modelling including erosion and deposition, Surv. Geophys., 24, 569-585.

Naaim, M., F. Naaim-Bouvet, and T. Faug (2004), Dense snow avalanche modeling: Flow, erosion, deposition, and obstacle effects, Cold Reg. Sci. Technol., 39, 193-204.

Norem, H., F. Irgens, and B. Schieldrop (1987), A continuum model for calculating snow avalanche velocities, IAHS Publ., 162, 363-379.

Perla, R., T. Cheng, and D. McClung (1980), A 2 parameter model of snowavalanche motion, J. Glaciol., 12(91), 197-207.

Perla, R., K. Lied, and K. Kristensen (1984), Particle simulation of snow avalanche motion, Cold Reg. Sci. Technol., 9, 191-202.

Platzer, K., P. Bartelt, and M. Kern (2007), Measurements of dense snow avalanche basal shear to normal stress ratios $(\mathrm{S} / \mathrm{N})$, Geophys. Res. Lett., 34, L07501, doi:10.1029/2006GL028670.

Pudasaini, S., and B. Domnik (2009), Energy considerations in accelerating rapid shear granular flows, Nonlin. Processes Geophys., 16(3), 399-407.

Pudasaini, S., and K. Hutter (2003), Rapid shear flows of dry granular masses down curved and twisted channels, J. Fluid Mech., 495, 193-208.

Pudasaini, S., and K. Hutter (2007), Avalanche Dynamics: Dynamics of Dense Granular Avalanches, 628 pp., Springer, New York.

Salm, B. (1966), Contribution to avalanche dynamics, IAHS Publ., 69, 221-226.

Salm, B. (1993), Flow, flow transition, and runout distances of flowing avalanches, Ann. Glaciol., 18, 221-226.

Salm, B., A. Burkard, and H. Gubler (1990), Berechnung von Fliesslawinen: Eine Anleitung für Praktiker mit Beispielen, Tech. Rep. 47, Eidg. Inst. Schnee- und Lawinenforschung Mitteilung, Davos, Switzerland.

Sampl, P., and T. Zwinger (2004), Avalanche simulation with SAMOS, Ann. Glaciol., 38, 393-398.

Savage, S. B., and K. Hutter (1989), The motion of a finite mass of granular material down a rough incline, J. Fluid Mech., 199, 177-215.

Savage, S. B., and K. Hutter (1991), The dynamics of avalanches of granular materials from initiation to run-out. Part I: Analysis, Acta Mech.86, 201-223.

Schaefer, M., L. Bugnion, M. Kern, and P. Bartelt (2010), Position dependent velocity profiles in granular avalanches, Granular Matter, 12(3), 327-336.

Sigrist, C., J. Schweizer, H. Schindler, and J. Dual (2006), The energy release rate of mode II fractures in layered snow samples, Int. J. Fract. $139(3-4), 461-475$

Simpson, J. (2010), Gravity Currents: In the Field and Laboratory, 242 pp., Cambridge Univ. Press, Cambridge, U. K. 
Son, R., J. Perez, and G. Voth (2008), Experimental measurements of the collapse of a two-dimensional granular gas under gravity, Phys. Rev. E, 78, 041302, doi:10.1103/PhysRevE.78.041302.

Sovilla, B., M. Schaer, M. Kern, and P. A. Bartelt (2008), Impact pressures and flow regimes in dense snow avalanches observed at the Vallee de la Sionne test site, J. Geophys. Res., 113, F01010, doi:10.1029/ 2006JF000688.

Sovilla, B., J. McElwaine, M. Schaer, and J. Vallet (2010), Variation of deposition depth with slope angle in snow avalanches: Measurements from Vallee de la Sionne, J. Geophys. Res., 115, F02016, doi:10.1029/ 2009JF001390.

Turnbull, B., J. McElwaine, and C. Ancey (2007), Kulikovskiy-SveshnikovaBeghin model of powder snow avalanches: Development and application, J. Geophys. Res., 112, F01004, doi:10.1029/2006JF000489.

Van Herwijnen, A., and J. Heierli (2009), Measurement of crack-face friction in collapsed weak snow layers, Geophys. Res. Lett., 36, L23502, doi:10.1029/2009GL040389.
Vilajosana, I., E. Surinach, G. Khazaradze, and P. Gauer (2007), Snow avalanche energy estimation from seismic signal analysis, Cold Reg. Sci. Technol., 50, 72-85.

Voellmy, A. (1955), Ueber die Zerstoerungskraft von Lawinen, Schweiz. Bauzeitung, 73(12/15/17/19), 159-162.

Voesselman, G., and H. G. Maas (2010), Airborne and Terrestrial Laserscanning, 320 pp., Whittles Publ., London.

Volk, G., and K. Kleemayr (1999), ELBA - Ein GIS-gekoppeltes Lawinensimulationsmodell, Anwendungen und Perspektiven, Oesterr. Z. für Vermessung und Geoinformation, 2, 84-92.

P. Bartelt, Y. Bühler, O. Buser, M. Christen, and L. Meier, WSL Institute for Snow and Avalanche Research SLF, Flüelastr. 11, CH-7260 Davos Dorf, Switzerland. (bartelt@slf.ch) 